\title{
Rice NAC transcription factor ONAC066 functions as a positive regulator of drought and oxidative stress response
}

\author{
Xi Yuan, Hui Wang, Jiating Cai, Yan Bi, Dayong Li and Fengming Song * (D)
}

\begin{abstract}
Background: NAC (NAM, ATAF and CUC) transcriptional factors constitute a large family with more than 150 members in rice and several members of this family have been demonstrated to play crucial roles in rice abiotic stress response. In the present study, we report the function of a novel stress-responsive NAC gene, ONAC066, in rice drought and oxidative stress tolerance.

Results: ONAC066 was localized in nuclei of cells when transiently expressed in Nicotiana benthamiana and is a transcription activator with the binding ability to NAC recognition sequence (NACRS) and AtJUB1 binding site (JBS). Expression of ONAC066 was significantly induced by PEG, $\mathrm{NaCl}, \mathrm{H}_{2} \mathrm{O}_{2}$ and abscisic acid (ABA). Overexpression of ONAC066 in transgenic rice improved drought and oxidative stress tolerance and increased ABA sensitivity, accompanied with decreased rate of water loss, increased contents of proline and soluble sugars, decreased accumulation of reactive oxygen species (ROS) and upregulated expression of stress-related genes under drought stress condition. By contrast, RNAi-mediated suppression of ONAC066 attenuated drought and oxidative stress tolerance and decreased ABA sensitivity, accompanied with increased rate of water loss, decreased contents of proline and soluble sugars, elevated accumulation of ROS and downregulated expression of stress-related genes under drought stress condition. Furthermore, yeast one hybrid and chromatin immunoprecipitation-PCR analyses revealed that ONAC066 bound directly to a JBS-like cis-elements in OsDREB2A promoter and activated the transcription of OSDREB2A.
\end{abstract}

Conclusion: ONAC066 is a nucleus-localized transcription activator that can respond to multiple abiotic stress factors. Functional analyses using overexpression and RNAi-mediated suppression transgenic lines demonstrate that ONAC066 is a positive regulator of drought and oxidative stress tolerance in rice.

Keywords: Drought tolerance, NAC transcription factor, ONAC066, Oxidative stress tolerance, Rice (Oryza sativa L,)

\section{Background}

During their lifespan, plants often suffer multiple environmental stresses including drought, salt and extreme temperatures, and thus they have evolved impressive mechanisms at molecular, biochemical, physiological, metabolic and developmental levels to cope with diverse environmental stresses [1-3]. At molecular level, a complicated signaling network is effectively and timely initiated upon perception of external stress signal, and this network ultimately reprograms the expression of a large

\footnotetext{
* Correspondence: fmsong@zju.edu.cn

National Key Laboratory for Rice Biology, Institute of Biotechnology, Zhejiang University, Hangzhou 310058, China
}

set of stress-responsive genes via a synergistic action of different types of transcription factors (TFs) in both temporal and spatial manners [1,3-5]. During the last decade, a number of members in TF families, e.g. NAC (NAM, AFAT and CUC), AP2/ERF, MYB, WRKY, bZIP, homeodomain, bHLH, NF-Y and CAMTA, have been characterized through knockout/knockdown and/or overexpression approaches and demonstrated to play important roles in plant abiotic stress response [6-12].

Among the stress-related TFs identified so far, the plant-specific NAC proteins are characterized by the presence of highly conserved NAC domains at Nterminal, which determine DNA-binding activity, and of variable domains at $\mathrm{C}$-terminal, which are responsible 
for transcription activity [13]. The NAC recognition sequence (NACRS), containing CATGT and CACG elements, from promoters of various downstream genes, was identified as a core DNA binding site of NAC TFs [14]. NAC TFs constitute a large family with 151 members in rice [15-17]. Recent extensive functional studies have demonstrated that NAC TFs play important roles in regulating biotic and abiotic stress responses in both model and crop plants $[8,12,18,19]$. Transcriptional profiling analysis revealed that a large portion of the rice ONAC family exhibited overlapping expression patterns in rice under various abiotic and biotic stresses [20,21]. Functional studies have identified at least 9 rice ONAC genes that play important roles in abiotic stress tolerance and these abiotic stress-related includes ONACO02 (SANC1/OsNAC9), ONAC048 (SNAC2/OsNAC6), ONACO09 (OsNAC5), ONAC122 (OsNAC10), ONAC045, ONAC058 (OsNAP), ONAC022, ONAC095 and ONACO03 (SNAC3) [22-34]. It was found that overexpression of SNAC1, SNAC2 or ONACO22 significantly enhanced tolerance to dehydration, cold and salt stresses in transgenic rice plants [22, 23], while transgenic rice plants overexpressing the root-specific OsNAC5, OsNAC6, OsNAC9, or OsNAC10 displayed significant improved drought tolerance [26, 27, 29, 35]. ONAC095 was found to have dual functions in drought and cold stress tolerance [34]. Whereas ONAC095 negatively regulates drought response, it oppositely acts as a positive regulator of cold response in rice [34]. In most of the cases, abscisic acid (ABA)-mediated signaling pathway [22, 23, 25, 29-31], stomatal movement and root system [22, 25, 27-29, 33] were found to be involved in the NAC-mediated improvement of abiotic stress tolerance in transgenic plants. However, SNAC3, which is one of the reported oxidative stress-related NAC TFs, functions as a positive regulator of drought, heat and oxidative stress response through ABA independent pathway [32]. Additionally, transgenic rice lines overexpressing some of the stress-related NAC TFs exhibited significant improvement of abiotic stress tolerance under severe stress conditions without any adverse effect on yield or even with yield increase [22, 26, 27, 29, 30], providing a promising potential for application of these stress-related NAC TFs in improvement of abiotic stress tolerance in crops [36, 37].

Arabidopsis NAC protein JUNGBRUNNEN1 (AtJUB1/ ANAC042) was found to function as a central regulator of growth and abiotic stress responses [38-40]. AtJUB1 regulates plant growth via directly repressing the expression of GA3ox1 and DWF4 genes encoding key enzymes of gibberellic acid and brassinosteroid biosynthesis and through activating the expression of DELLA genes such as GA Insensitive and RGA-like 1 [41, 42]. Overexpression of AtJUB1 in Arabidopsis or MusaNAC042 (banana homologue of AtJUB1) in banana resulted in enhanced tolerance to various abiotic stress including drought stress, whereas knockdown of Arabidopsis AtJUB1 or silencing of tomato SlJUB1 (tomato homologue of AtJUB1) led to decreased tolerance to drought and oxidative stress [38, 39, 43-45]. It was shown that, in regulating the abiotic stress response, the Arabidopsis AtJUB1 and tomato SIJUB1 can directly bind to the promoters of AtDREB2A, SIDREB1 and SIDREB2, respectively, which encode AP2-type TFs known to be critical regulators of abiotic stress response [38, 39]. Besides, AtJUB1 acts downstream of the HD-Zip class I TF AtHB13, which is a positive regulator of drought tolerance, establishing a joint drought stress control module between AtHB13 and AtJUB1 [43].

We were interested in the biological functions of rice $O N A C$ genes in abiotic and biotic stress response and we previously identified through bioinformatics analysis of publicly available microarray data a number of stressresponsive ONAC genes in rice response to biotic and abiotic stresses [20]. Among these stress-responsive ONAC genes, ONACO22 and ONAC095 were previously shown to play roles in drought, salt and cold stress response [33, 34]. In the present study, we explored the function of ONAC066, the rice homologue of AtJUB1, in regulating stress response using overexpression and RNA interference (RNAi)-mediated suppression transgenic lines. We found that overexpression of ONAC066 in transgenic rice improved drought and oxidative stress tolerance while RNAi-mediated suppression of ONACO66 attenuated drought and oxidative stress tolerance. We also showed that ONAC066 bound directly to a JBS-like (JBSL) cis-element in OsDREB2A promoter and thereby activated transcription of OsDREB2A. Our data demonstrate that ONAC066 is a positive regulator of drought and oxidative stress tolerance in rice.

\section{Results}

ONAC066 is a putative stress-responsive NAC gene in rice The ONAC066 gene (LOC_Os03g56580) contains a complete ORF of $1089 \mathrm{bp}$, which encodes a polypeptide of 362 amino acid (aa) with a calculated molecular weight of $40.6 \mathrm{kDa}$ and $p \mathrm{I}$ of 6.4 . The ONAC066 protein harbors a single typical conserved NAC domain, spanning 120 aa between 51 aa and 170 aa, at $\mathrm{N}$-terminus, which can be further divided into 5 subdomains (A to E) (Additional file 1: Figure S1A). Phylogenetic tree analysis suggests that ONAC066 belongs to Phylogeny Group IV [16], and is closely related to ONAC096, ONAC140 and ANAC042/AtJUB1 (Additional file 1: Figure S1B), showing $47.7,37.14$ and $34.81 \%$ of identity at amino acid level, respectively. Bioinformatics analysis at PlantCARE [46] indicates that the promoter region (1.5 Kb upstream of ATG) of the ONAC066 gene contains several putative stress-related cis- elements, including two ABREs (ABAresponsive element involved in $\mathrm{ABA}$ responsiveness, [47]), one CGTCA motif (a cis-element involved in JA 
responsiveness, [48]), one GCC box (a cis-element involved in elicitation, wounding and pathogen responsiveness, [49]), two TCA elements (a cis-element involved in SA responsiveness, $[50,51])$, two TC-rich elements (a cis-element involved in defense and stress responsiveness, [52]), and two HSEs (a cis-element involved in heat stress responsiveness, [53]) (Additional file 1: Figure $\mathrm{S1C})$. The presence of these stress-responsive cis-elements in its promoter suggests that ONACO66 may be responsive to multiple abiotic stress cues.

\section{DNA binding and transactivation activities of ONAC066}

To determine the TF activity of ONAC066 and, if any, the region in ONAC066 responsible for TF activity, we examined using $\mathrm{Y} 1 \mathrm{H}$ assays the transactivation activity of entire ONAC066 and a series of truncated variants including ONAC066-N (1-170 aa), ONAC066-C1 (171362 aа), ONAC066-C2 (171-264 aa) and ONAC066-C3 (265-362 aa) (Fig. 1a, left). Yeast transformants harboring pGBKT7-ONAC066 or one of these variants grew well in SD/-Trp medium (Fig. 1a, right). Whereas transformants harboring pGBKT7-ONAC066-N and pGBKT7ONAC066-C2 failed to grow on SD/-Trp-His/5 mM 3-AT medium, transformants harboring pGBKT7-ONAC066 and variants pGBKT7-ONAC066-C1 and pGBKT7-ONAC066$\mathrm{C} 3$ did grow and show $\beta$-galactosidase activity (Fig. 1a, right). These results indicate that ONAC066 has a transactivation activity and the region of $265-362$ aa at Cterminus is critical for the transactivation activity.

To explore the DNA binding activity of ONAC066, we examined the binding capacity of ONAC066 to NACRS, a canonical NAC core binding sequence [14], and JBS, a novel NAC binding sequence [39], using Y1H assays. For this, tandem repeated $3 \times$ NACRS and $3 \times$ JBS were constructed into pHis2 vector and co-transformed with Rec2-ONAC066 into yeast cells (Fig. 1b). Yeast cells cotransformed with Rec2-ONAC066 and pHis2-3× NACRS or pHis $2-3 \times \mathrm{JBS}$ grew normally while yeast cells co-transformed with pGADT7-Rec2 empty vector and pHis2-3 $\times$ NACRS or pHis2-3 $\times$ JBS failed to grow on SD/-Trp-Leu-His medium with $50 \mathrm{mM}$ or $100 \mathrm{mM}$ 3-AT (Fig. 1c). These results indicate that ONAC066 has DNA binding activity and can bind to both the NAC binding sequences, NACRS and JBS.

\section{Nucleus localization of ONAC066}

The subcellular localization of ONAC066 was examined through transient expression of a GFP-tagged ONAC066 construct in leaves of Nicotiana benthamiana plants expressing a red nuclear marker RFP-H2B protein [54]. At $48 \mathrm{~h}$ after agroinfiltration, the ONAC066::GFP fusion was microscopically observed to be solely localized in nucleus, which was co-localized with the known nuclear marker RFP-H2B protein (Fig. 1d). In contrast, GFP alone was seen to be ubiquitously distributed throughout the cell without specific localization (Fig. 1d). These results indicate that ONAC066 is a nucleus-localized protein.

\section{Expression patterns of ONAC066 upon abiotic stress treatment}

ONAC066 is expressed in all tissues, especially in leaves and seeds, as examined using public microarray data at NCBI (http://www.ncbi.nlm.nih.gov/geo) under accession number GSE6893 (Additional file 2: Figure S2). We examined by quantitative real time-PCR (qRT-PCR) the responsiveness of ONAC066 to abiotic stresses and exogenous ABA. The expression level of ONAC066 in PEG6000-treated plants started to increase at $1 \mathrm{~h}$ after treatment (hpt) and increased gradually over a period of $24 \mathrm{~h}$, with a peak at $24 \mathrm{hpt}$, showing 35 -fold of increase over that in control plants (Fig. 2a). High levels of ONAC066 expression were observed in $\mathrm{NaCl}$-treated plants at 1 and $12 \mathrm{hpt}$, showing 6.8- and 5.3-fold of increase over that in control plants (Fig. 2b). In $\mathrm{H}_{2} \mathrm{O}_{2}$-treated plants, significant induction of ONACO66 expression was detected at 12 and $24 \mathrm{hpt}$, leading to 4.8 - and 18-fold of increased over those in control plants (Fig. 2c). In ABA-treated plants, the expression level of ONACO66 showed significant increase only at $12 \mathrm{hpt}$, giving a 3.6-fold increase over that in control plants (Fig. 2d). These results indicate that ONAC066 is an abiotic stress- and ABA-responsive rice ONAC gene.

\section{Generation of ONAC066-OE and ONAC066-RNAi transgenic lines}

To investigate the function of ONACO66, we generated ONAC066-OE and ONAC066-RNAi transgenic rice lines in japonica rice cv. Zhonghua11 (ZH11) background (Additional file 3: Figure S3A). qRT-PCR revealed that the transcript levels of ONACO66 in ONAC066-OE lines OE11 and OE12 showed 57.1- and 79.9-fold higher than that in wild type (WT) plants, while the levels in ONAC066-RNAi lines Ri1 and Ri21 were estimated to be 34 and $39 \%$ of the level in WT plants (Additional file 3: Figure S3B). The ONAC066GFP fusion in ONAC066-OE lines OE11 and OE12 plants was detected with GFP antibody, showing a band of $\sim 67.8 \mathrm{kD}$ that matched the molecular weight of the fusion (Additional file 3: Figure S3C). No significant defect in morphological, growth and developmental characters was observed in ONAC066-OE and ONAC066RNAi plants.

\section{Overexpression of ONAC066 improved while suppression of ONAC066 weakened drought tolerance}

We first explored whether ONACO66 played a role in drought tolerance by phenotyping ONAC066-OE and ONAC066-RNAi plants under drought condition and comparing with WT plants. Under normally watered 
A

ONAC066-N (1-170)

1

NAC

170

264

362

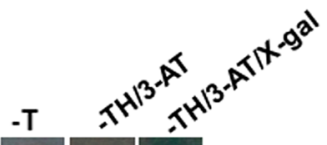

ONAC066-C1 (171-362)

ONAC066-C2 (171-264)

ONAC066-C3 (265-362)

\section{NAC}

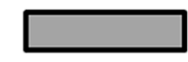

Negative control

Positive control

B

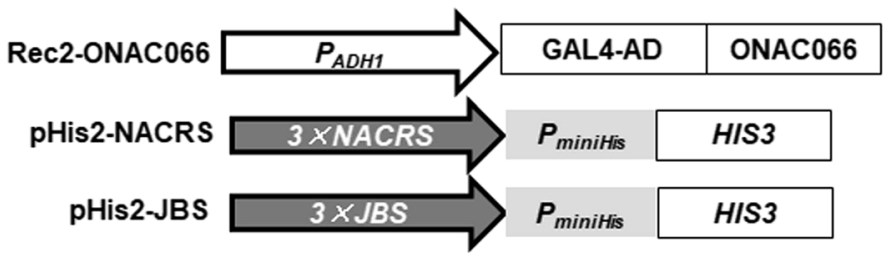

C

$-\mathrm{TL}$

-TLH/50mM 3-AT -TLH/100mM 3-AT
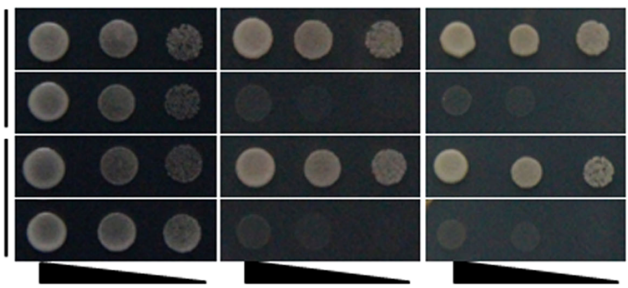

Rec2-ONAC066

Rec2

pHis2-JBS

GFP

RFP

Merged

Rec2-ONAC066

D
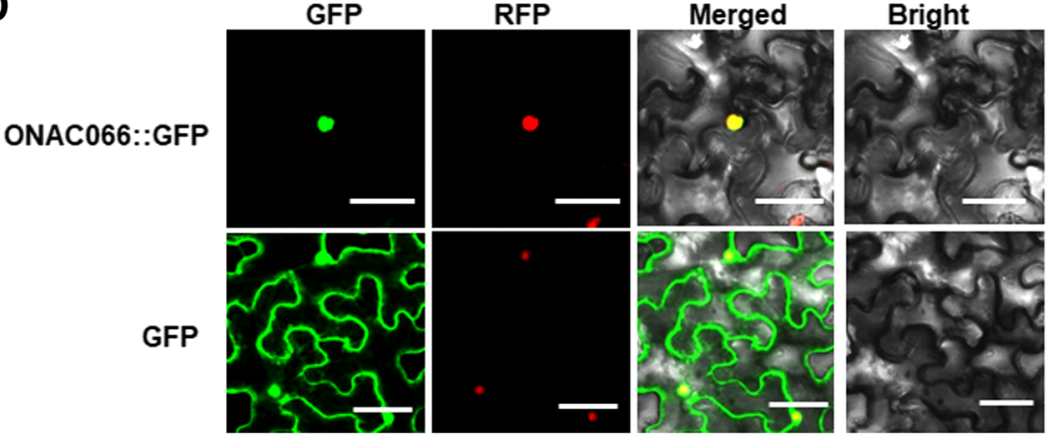

Rec2

Fig. 1 Transactivation activity and subcellular localization of ONAC066. a ONAC066 has transactivation activity. Full length and truncated mutants of ONAC066 were fused to GAL4-binding domain and transformed into yeasts. Yeast cells harboring each of the constructs, pGBKT7 empty vector (negative control) or pGBKT7-ONAC022 (positive control) were streaked on medium plates of SD/-Trp, SD/-Trp-His/3-AT and SD/-Trp-His/3-AT/Xa-gal for 3 days at $28^{\circ} \mathrm{C}$. $\mathbf{b}$ Schematic diagram of different constructs used in ONAC066-NACRS or ONAC066-JBS binding assays. c ONAC066 binds to NACRS and JBS in vivo. The Rec2-ONAC066 and Rec2 empty vectors were co-transformed with the reporter constructs pHis-NACRS or pHis-JBS into yeasts and co-transformed yeast cells were streaked by a series of 10-fold dilutions on plates of SD/-Trp-Leu, SD/-Trp-Leu-His, SD/-Trp-LeuHis/50 mM 3-AT and SD/-Trp-Leu-His/100 mM 3-AT. d ONAC066 is localized in nucleus. Agrobacteria harboring pCAMBIA1300-ONAC066-GFP or pCAMBIA1300-GFP were infiltrated into leaves of $\mathrm{N}$. benthamiana plants expressing a red nucleus marker protein H2B-RFP. Leaf samples were collected at $48 \mathrm{~h}$ after agroinfiltration. Microscopic examination was performed under a confocal laser scanning microscope in dark field for green fluorescence (left), red fluorescence (middle left), white field for cell morphology (middle right) and in combination (right), respectively. Bar $=10 \mu m$. Experiments in (a), (c) and (d) were repeated for three times with similar results 

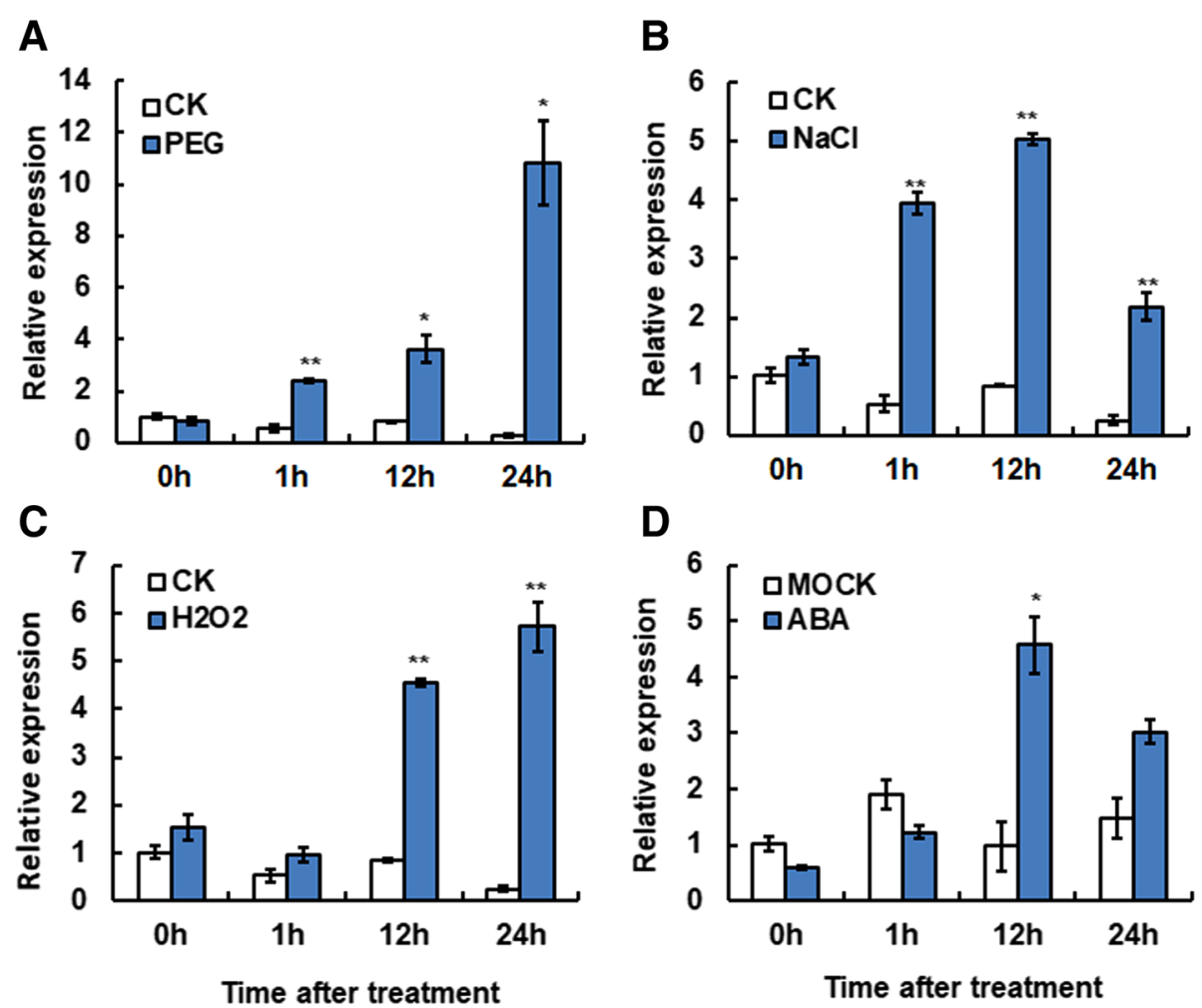

Fig. 2 Stress-inducible expression of ONAC066. Three-week-old seedlings were exposed to (a) 20\% PEG6000, b $100 \mathrm{mM} \mathrm{NaCl}, \mathbf{c} 100 \mathrm{mM} \mathrm{H} \mathrm{O}_{2}$ or (d) $50 \mu \mathrm{M}$ ABA. Samples were collected at different time intervals and the transcript level of ONAC066 was quantified by qRT-PCR. Relative expression level of ONAC066 was normalized to the internal control of 18 s-rRNA gene. Data presented are the means \pm SD from three independent experiments

condition, the growth phenotype of ONAC066-OE and ONAC066-RNAi plants were indistinguishable from WT plants (Fig. 3a, upper panel). At 7 days after drought treatment by water withholding, the ONAC066-RNAi plants showed earlier and severer drought symptom, represented by rolled leaves and wilted plants, while the ONAC066-OE plants displayed relatively later and slighter drought symptom, as compared with the WT plants (Fig. 3a, middle panel). At 2 days after re-watering, 79 and 53\% of ONAC066-OE lines OE11 and OE12 plants recovered and survived, showing 36 and 20\% higher over those in WT plant (43 and 33\%) (Fig. 3a, lower panel). By contrast, only $14 \%$ of the ONAC066-RNAi line Ri21 plants and none of the Ri1 plants recovered and survived, showing 26 and $43 \%$ lower over those in WT plant (40 and 43\%) (Fig. 3a, lower panel). These results indicated that overexpression of ONACO66 improved while suppression of ONACO66 weakened drought tolerance in transgenic rice.

To further confirm the involvement of ONAC066 in drought tolerance, we compared water status, as reflected by water loss rate (WLR) and relative water content (RWC), in ONAC066-OE and ONAC066-RNAi plants with those in WT plants after drought stress treatment. As compared to those in WT plants, WLR in drought stress-treated ONAC066-OE plants was significantly reduced by $4-8 \%$, while WLR in drought stresstreated ONAC066-RNAi plants was increased by $5-9 \%$ at 4, 6, 8 and $10 \mathrm{~d}$ after treatment, respectively (Fig. 3b). As consequences, the drought stress-treated ONAC066OE plants had significantly higher RWC, showing 8$12 \%$ of increase, while the drought stress-treated ONAC066-RNAi line Ri1 plants showed lower RWC, giving $5-10 \%$ of decrease, as compared to those in drought stress-treated WT plants, at 4, 6, 8 and $10 \mathrm{~d}$ after treatment (Fig. 3c). These results further confirm that ONAC066 plays a significant role in rice drought stress tolerance.

Overexpression of ONACO66 enhanced while suppression of ONAC066 attenuated oxidative stress tolerance

It was reported that overexpression of AtJUB1, an Arabidopsis homologue of ONAC066 (Additional file 1: Figure $\mathrm{S} 1 \mathrm{~B}$ ), conferred tolerance to exogenous $\mathrm{H}_{2} \mathrm{O}_{2}$ [39]. We thus examined whether ONAC066 played a role in rice oxidative stress tolerance. Without $\mathrm{H}_{2} \mathrm{O}_{2}$ treatment, no significant phenotype appeared on leaf discs from 3week-old ONAC066-OE, ONAC066-RNAi and WT plants during a period of $48 \mathrm{~h}$ in the $\mathrm{H}_{2} \mathrm{O}_{2}$ tolerance assays (Fig. 4a). At $48 \mathrm{~h}$ after treatment in $\mathrm{H}_{2} \mathrm{O}_{2}$ solution 


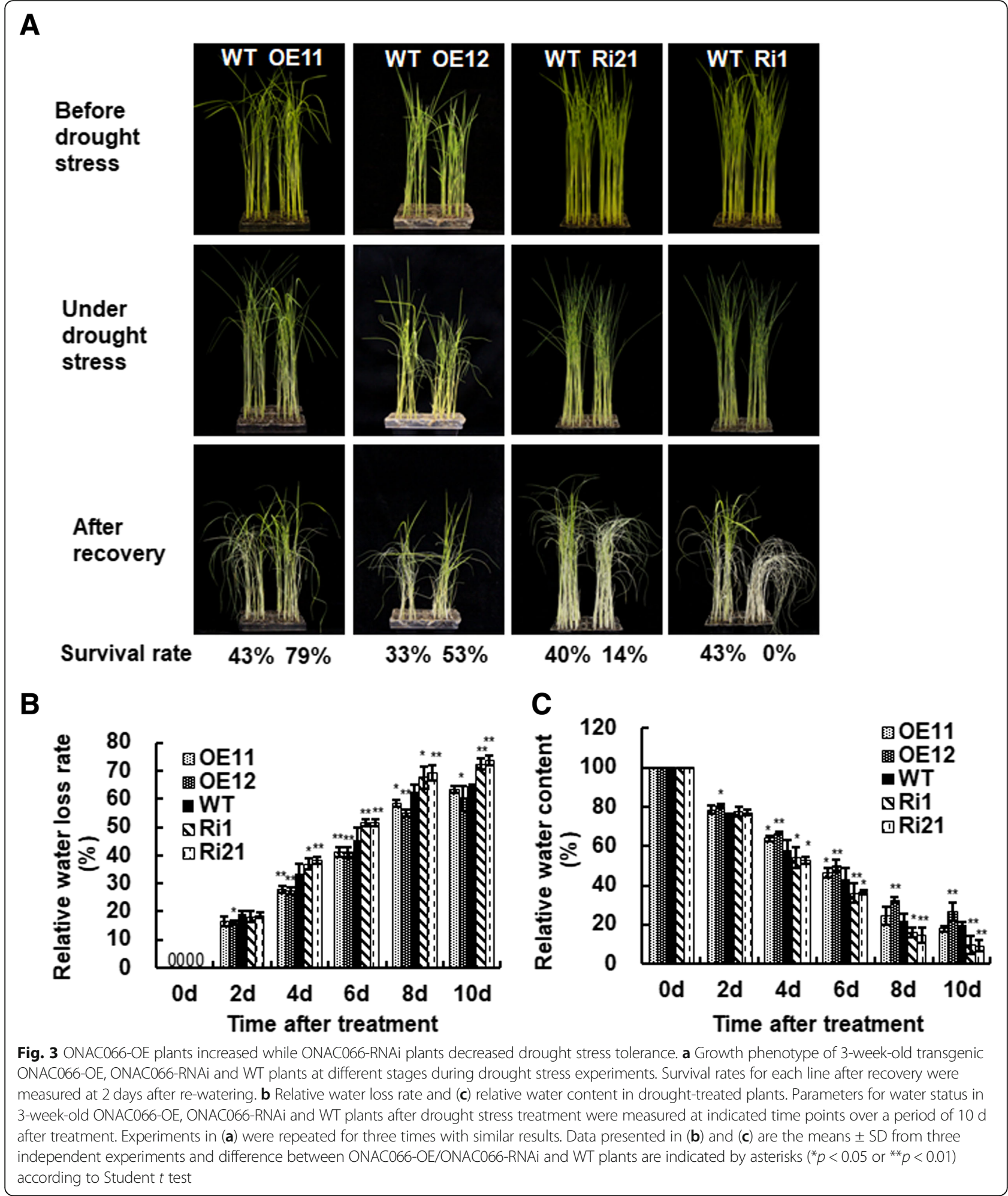

at concentrations of $0.5,1.0$ and $1.5 \%$, yellowing and chlorosis symptoms were observed in leaf discs from ONAC066-OE, ONAC066-RNAi and WT plants (Fig. 4a); however, yellowing and chlorosis symptoms in leaf discs from ONAC066-OE plants were less severe while these symptoms in leaf discs from ONAC066RNAi plants were much evident, as compared with those in WT plants (Fig. 4a). These observations were confirmed by measuring chlorophyll contents in leaf discs from ONAC066-OE, ONAC066-RNAi and WT plants 


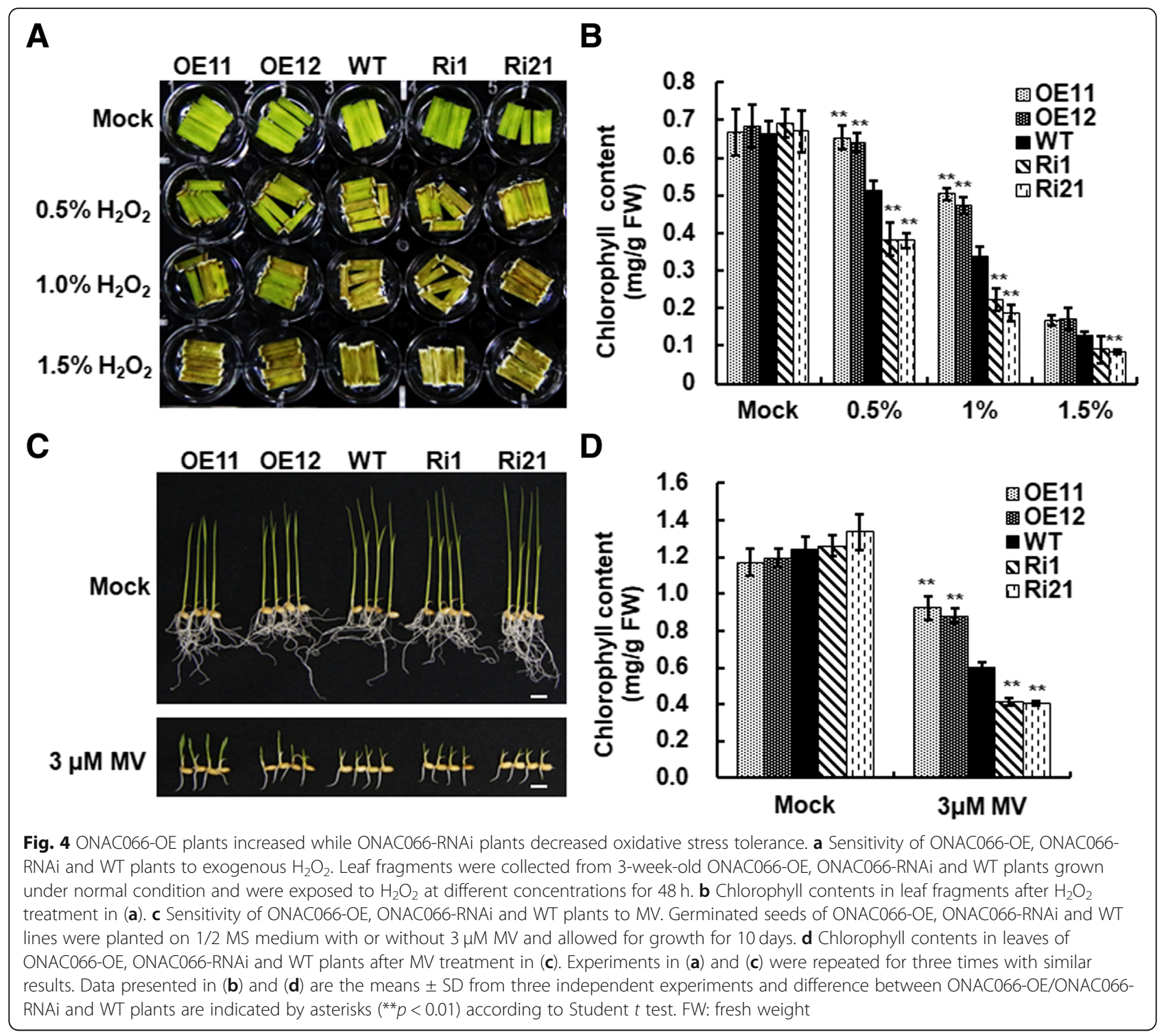

after $\mathrm{H}_{2} \mathrm{O}_{2}$ treatments (Fig. 4b). Without $\mathrm{H}_{2} \mathrm{O}_{2}$ treatment, chlorophyll contents in leaf discs from ONAC066-OE and ONAC066-RNAi plants were comparable to that in WT plant (Fig. 4b). However, chlorophyll contents in leaf discs from ONAC066-RNAi lines Ri1 and Ri21 plants were dramatically decreased, leading to $25-26 \%, 34-45 \%$ and $29-35 \%$ of reduction, as compare with those in WT plants, at $48 \mathrm{~h}$ after treatment in $0.5,1.0 \%$ or $1.5 \% \mathrm{H}_{2} \mathrm{O}_{2}$ solution, respectively (Fig. $4 \mathrm{~b}$ ). By contrast, chlorophyll contents in leaf discs from ONAC066-OE lines OE11 and OE12 plants were significantly higher, showing $25-27 \%$ and $40-49 \%$ of increase, as compare with those in WT plants, at $48 \mathrm{~h}$ after treatment in $0.5 \%$ or $1.0 \% \mathrm{H}_{2} \mathrm{O}_{2}$ solution, respectively (Fig. 4b). These results indicate that overexpression of ONAC066 increased while suppression of ONAC066 attenuated the tolerance to exogenous $\mathrm{H}_{2} \mathrm{O}_{2}$.
We further explored whether modification of ONAC066 affected the tolerance of transgenic rice plants to methyl viologen (MV) (a compound generating reactive oxygen species including superoxide anion), by comparing growth phenotype of and chlorophyll content in ONAC066-OE and ONAC066-RNAi seedlings grown on 1/2 Murashige and Skoog (MS) medium supplemented with MV to those of WT seedlings. In 1/2 MS medium without supplementation of MV, slight suppression or promotion in growth of ONAC066-OE and ONAC066RNAi seedlings, respectively, were observed, as compared with that of WT seedlings (Fig. 4c). As compared with growth of the seedlings grown MV-free 1/2 MS, growth of ONAC066-OE, ONAC066-RNAi and WT seedlings grown on $1 / 2 \mathrm{MS}$ supplemented with $3 \mu \mathrm{M}$ MV were significantly suppressed (Fig. 4c). On medium with MV, ONAC066-OE seedlings showed less growth 
suppression while ONAC066-RNAi seedlings exhibited severer growth suppression, as compared to WT seedlings (Fig. 4c). Similarly, slight increase or decrease in chlorophyll contents in ONAC066-OE and ONAC066RNAi seedlings, respectively, were observed, as compared with that in WT seedlings, when grown in 1/2MS medium without supplementation of MV (Fig. 4d). However, when grown on medium with $\mathrm{MV}$, chlorophyll contents in ONAC066-OE, ONAC066-RNAi and WT seedlings were significantly decreased, as compared with that in seedlings grown in MV-free medium (Fig. 4d). Chlorophyll contents in ONAC066-OE lines OE11 and OE12 seedlings showed $47-55 \%$ higher while those in ONAC066-RNAi lines Ri1 and Ri21 seedlings exhibited $31-32 \%$ lower than that in WT seedlings (Fig. 4d). These results indicate that overexpression of ONAC066 increased while suppression of ONACO66 attenuated the tolerance to MV.

\section{Overexpression of ONAC066 increased while suppression} of ONAC066 decreased ABA sensitivity

The fact that expression of ONAC066 was induced by ABA led us to explore whether altered expression of ONAC066 affected ABA sensitivity in ONAC066-OE and ONAC066-RNAi plants. We examined the ABA sensitivity of ONAC066-OE and ONAC066-RNAi seedlings and compared with WT seedlings by analyzing seedling growth in the presence of ABA. Growth of ONAC066-OE, ONAC066-RNAi seedlings was indistinguishable from WT seedlings in the absence of ABA, but their growth was suppressed when grown on $1 / 2 \mathrm{MS}$ supplemented with ABA (Fig. 5a). However, growth of ONAC066-OE seedlings was severely suppressed while ONAC066-RNAi seedlings was slightly suppressed, as compared with that of WT seedlings, when grown on 1/ 2 MS supplemented with $2 \mu \mathrm{M}$ or $5 \mu \mathrm{M}$ ABA (Fig. $5 \mathrm{a}$ ). Root length of ONAC066-OE lines OE11 and OE12 seedlings was significantly shorted, resulting in $32-41 \%$ and $47-59 \%$ of reduction, while root length of ONAC066-RNAi lines Ri1 and Ri21 seedlings were longer, leading to $25-31 \%$ and $31-45 \%$, respectively, than those in WT seedlings, when grown on $1 / 2$ MS supplemented with $2 \mu \mathrm{M}$ or $5 \mu \mathrm{M}$ ABA (Fig. $5 \mathrm{~b}$ ). By contrast, on ABA-containing 1/2 MS, shoot length of ONAC066$\mathrm{OE}$ and ONAC066-RNAi seedlings were comparable to that of WT seedlings, except for a significant reduction in shoot length of ONAC066-OE line OE12 seedlings on $1 / 2$ MS containing $2 \mu \mathrm{M}$ ABA (Fig. $5 \mathrm{c}$ ). These results

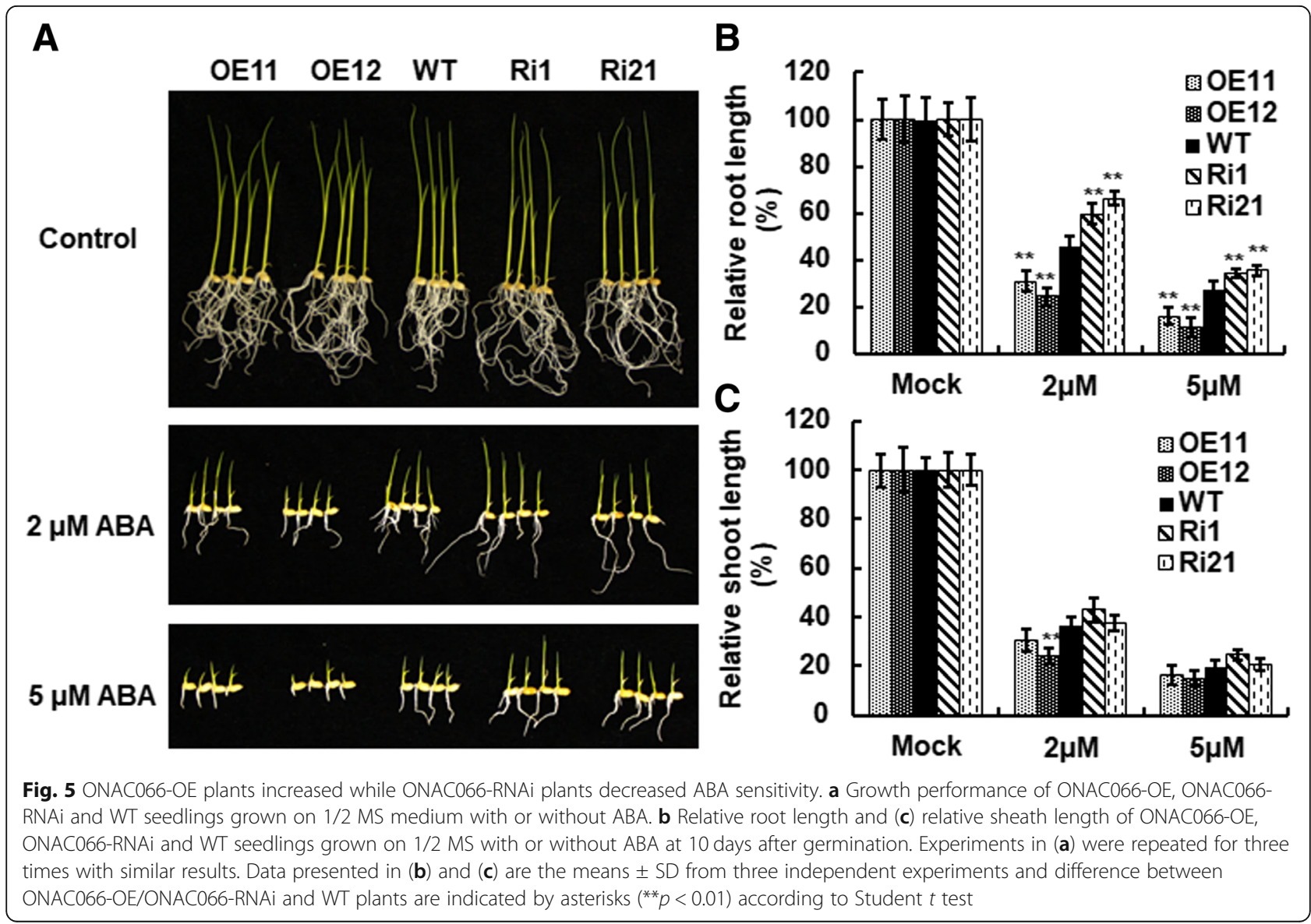


indicate that overexpression of ONAC066 increased while suppression of ONAC066 decreased ABA sensitivity.

Altered expression of ONACO66 affected the accumulation of stress-related metabolites during drought stress response To explore the possible physiological basis of ONAC066mediated stress response, we analyzed and compared the changes in some stress-related metabolites such as proline and soluble sugars in ONAC066-OE and ONAC066-RNAi plants with those in WT plants under normal watering and water withholding drought conditions. Under normally watered condition, contents of proline and soluble sugars in both ONAC066-OE and ONAC066-RNAi plants were comparable to those in WT plants (Fig. 6a and b). At 5 days after water withholding drought treatment, contents of proline and soluble sugars were increased in ONAC066-OE, ONAC066-RNAi and WT plants as compared with those in their corresponding control plants grown under normal watering condition (Fig. 6a and b). The ONAC066-OE lines OE11 and OE12 plants showed higher contents of proline and soluble sugars, leading to $34-52 \%$ and $18-42 \%$ of increase, respectively, while the ONAC066-RNAi lines Ri1 and Ri21 plants exhibited lower contents of proline and soluble sugars, giving $26-40 \%$ and $37-45 \%$ of decrease, respectively, as compared with those in WT plants, under water withholding drought condition (Fig. 6a and b). These results indicate that overexpression of ONACO66 stimulated while suppression of ONACO66 decreased the accumulation of stress-related metabolites such as proline and soluble sugars during drought stress response.

ROS is often involved in abiotic stress response by causing oxidative damage. We thus analyzed and compared the accumulation of ROS in ONAC066-OE and ONAC066-RNAi plants with those in WT plants under normally watering and water withholding drought conditions to explore the involvement of ROS in ONAC066mediated drought stress response. Qualitative staining

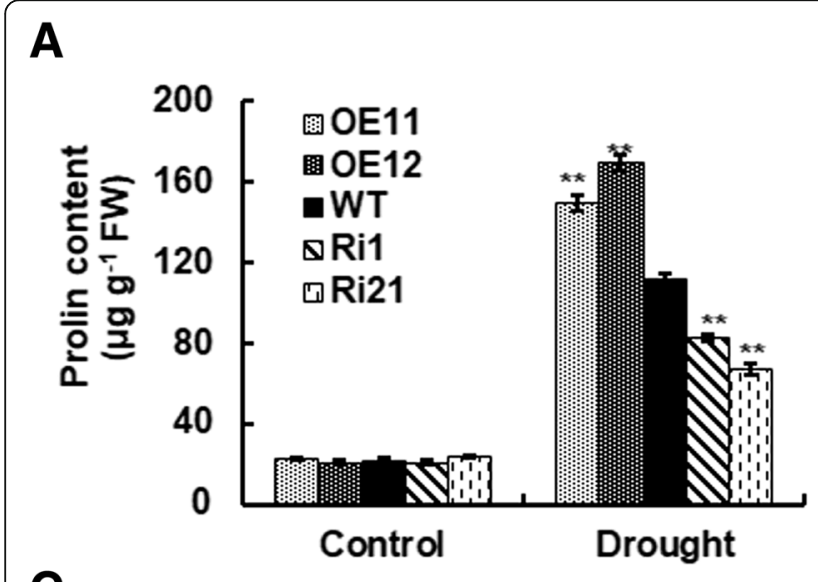

B
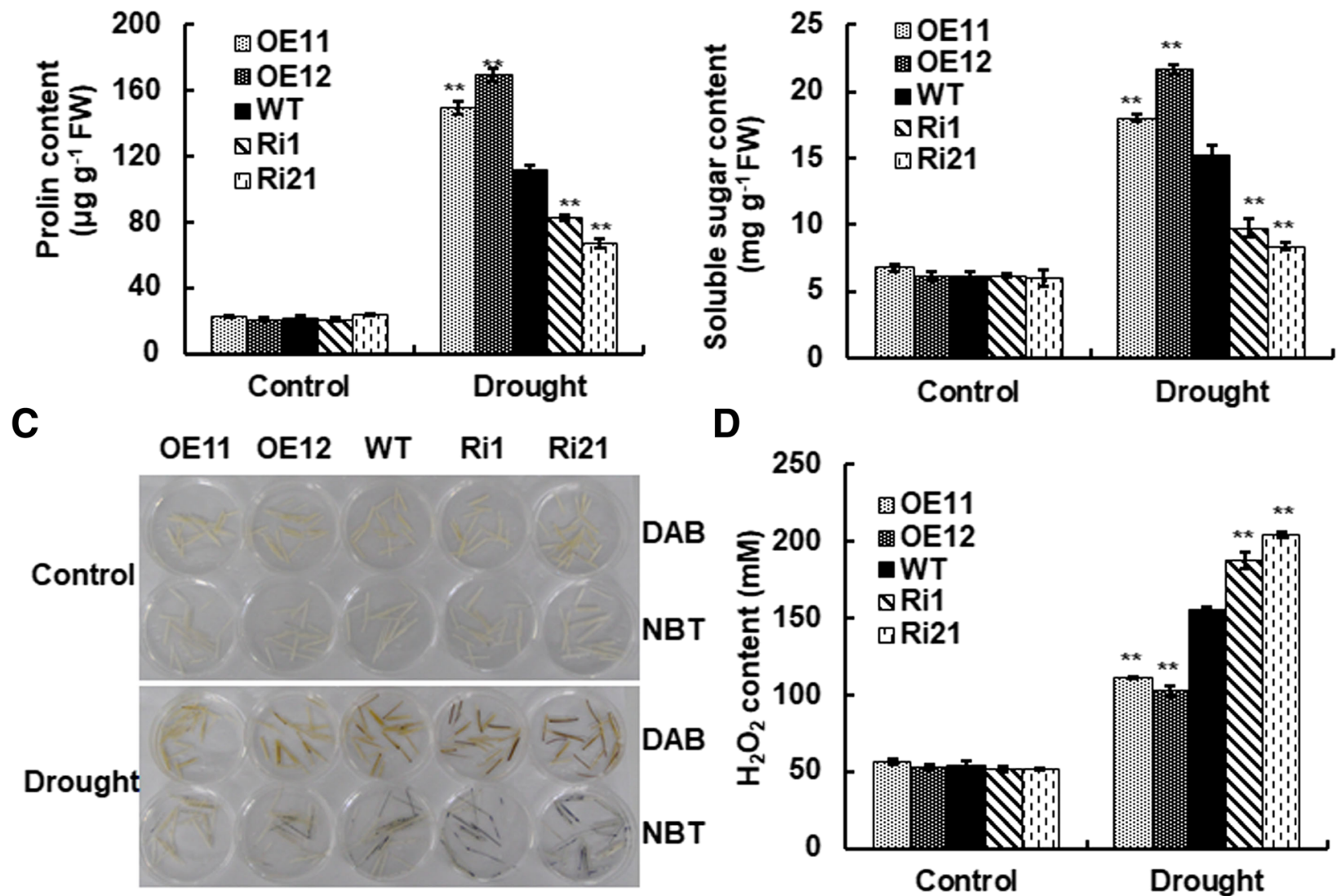

D

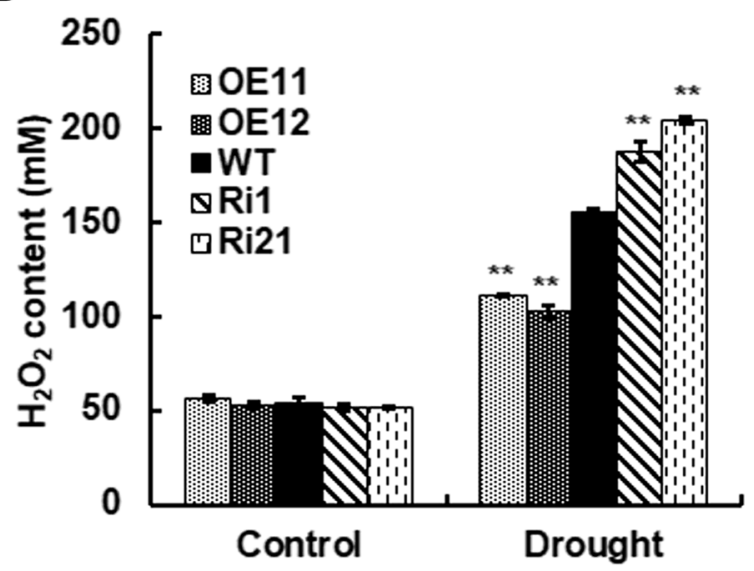

Fig. 6 Drought-induced accumulation of proline, soluble and ROS in ONACO66-OE and ONAC066-RNAi plants. a Proline content and (b) soluble sugar content. $\mathbf{c}$ In situ detection of $\mathrm{H}_{2} \mathrm{O}_{2}$ and superoxide anion in leaves by DAB and NBT staining, respectively. $\mathbf{d} \mathrm{H}_{2} \mathrm{O}_{2}$ levels in leaves. Leaf samples were collected from 3-week-old ONAC066-OE, ONAC066-RNAi and WT plants grown under normally watered and drought-stressed (at 5 days after water withholding) conditions and were subjected to physiological measurements. Experiments in (c) were repeated for three times with similar results. Data presented in $(\mathbf{a}),(\mathbf{b})$ and $(\mathbf{d})$ are the means \pm SD from three independent experiments and significant difference between ONAC066-OE/ONAC066RNAi and WT plants under the same treatment are indicated by asterisks $\left({ }^{* *} p<0.01\right.$ and $\left.{ }^{*} p<0.05\right)$ according to Student $t$ test. FW: fresh weight 
by $3,3^{\prime}$-diaminobenzidine (DAB) and nitroblue tetrazolium (NBT) and quantitative measurement indicated that staining of $\mathrm{H}_{2} \mathrm{O}_{2}$ and superoxide anion and accumulation of $\mathrm{H}_{2} \mathrm{O}_{2}$ in leaf tissues of ONAC066-OE and ONAC066-RNAi plants were indistinguishable from those in WT plants grown under normally watering condition (Fig. $6 \mathrm{c}$ and d). Staining of $\mathrm{H}_{2} \mathrm{O}_{2}$ and superoxide anion was much evident and the accumulation levels of $\mathrm{H}_{2} \mathrm{O}_{2}$ were increased in leaf tissues of ONAC066-OE, ONAC066-RNAi and WT plants than those in their corresponding control plants grown under normally watering condition (Fig. $6 \mathrm{c}$ and d). At 5 days after water withholding drought treatment, staining of $\mathrm{H}_{2} \mathrm{O}_{2}$ and superoxide anion in leaf tissues of ONAC066-OE plants was less evident while the staining in leaf tissues of ONAC066-RNAi plants was much obvious, as compared with those in WT plants (Fig. 6c). Accordingly, the ONAC066-OE lines OE11 and OE12 plants showed higher $\mathrm{H}_{2} \mathrm{O}_{2}$ accumulation level, leading to $28-34 \%$ of reduction, while the ONAC066-RNAi lines Ri1 and Ri21 plants exhibited lower contents of proline and soluble sugars, giving $21-31 \%$ of increase, as compared with those in WT plants, under water withholding drought condition (Fig. 6d). These results indicate that overexpression of ONAC066 suppressed while suppression of ONAC066 stimulated the accumulation of ROS during drought stress response.

\section{Altered expression of ONACO66 affected the expression of drought-responsive and ROS scavenging genes}

To gain further insight into the possible molecular mechanism of ONAC066-meditated stress response, we analyzed and compared the expression changes of several drought-responsive genes in ONAC066-OE and ONAC066-RNAi plants with those in WT plants under normally watering and water withholding drought conditions. Under normal watering condition, the expression levels of OsDREB2A [55], OsERD1 (a homolog of Arabidopsis AtERD1 [56]), OsLEA3 [57], OsP5CS1 [58], and OsbZIP23 [59] in ONAC066-OE and ONAC066-RNAi plants were comparable to those in WT plants (Fig. 7). Under water withholding drought condition, the expression levels of these drought responsive genes in ONAC066-OE and ONAC066-RNAi plants were significantly increased, as compared with those in their corresponding control plants grown under normally watering condition (Fig. 7). At 5 days after water withholding drought treatment, the expression levels of OsDREB2A, OsERD1, OsLEA3, OsP5CS1 and OsbZIP23 in ONAC066OE lines OE11 and OE12 plants showed 1.0-2.1, 1.7-2.3, $0.7-1.3,1.8-4.5$ and $0.9-1.8$ folds higher, while the expression levels in ONAC066-RNAi lines Ri1 and Ri21 plants exhibited $35-54 \%, 36-65 \%, 27-33 \%, 68-87 \%$ and $13-81 \%$ lower, as compared with those in WT plants, under water withholding drought condition (Fig. 7). These results indicate that overexpression of ONAC066 upregulated while suppression of ONAC066 downregulated the expression of drought-responsive genes during drought stress response.

We also analyzed and compared the expression changes of some ROS generating and scavenging-related genes, including RbohF (NADPH oxidase, LOC_Os08g35210), POD (peroxidase, LOC_Os01g73200), CATA (catalase, LOC_ Os02g02400) and APX8 (ascorbate peroxidase, LOC_ Os02g34810) [32], in ONAC066-OE and ONAC066-RNAi plants with those in WT plants under normally watering and water withholding drought conditions. Expression of RbohF in ONAC066-OE and ONAC066-RNAi plants under normally watering condition and in ONAC066-RNAi plants under water withholding drought condition was comparable to those in WT plants; however, its expression in ONAC066-OE plants under water withholding drought condition was significantly increased, showing $1.0-1.3$ folds of increase, as compared with that in WT plants (Fig. 7). Under normally watering condition, the expression levels of POD, CATA and APX8 in ONAC066-OE lines OE11 and OE12 plants were to be $1.5-2.4,1.2-1.0$ and $0.3-0.8$ folds higher, respectively, while the expression levels in ONAC066-RNAi lines Ri1 and Ri21 plants showed 39-61\%, 63-84\% and 23-70\% lower, respectively, than those in WT plants (Fig. 7). Under water withholding drought condition, the expression levels of these ROS scavenging genes in ONAC066-OE and ONAC066-RNAi plants were significantly increased, as compared with those in their corresponding control plants grown under normally watering condition (Fig. 7). At 5 days after water withholding drought treatment, the expression levels of POD, CATA and APX8 in ONAC066-OE lines OE11 and OE12 plants were markedly upregulated by $0.5-1.2,0.4-0.8$ and $0.6-0.7$ folds, respectively, while the expression levels in ONAC066-RNAi lines Ri1 and Ri21 plants were downregulated by $78-80 \%$, 40 $49 \%$ and $13-32 \%$, respectively, as compared with those in WT plants, under water withholding drought condition (Fig. 7). These results indicate that overexpression of ONAC066 upregulated while suppression of ONAC066 downregulated the expression of ROS scavenging genes in transgenic rice under normal growth condition and during drought stress response.

\section{ONAC066 bound to a JBSL cis-element in OsDREB2A promoter}

The binding of ONAC066 to JBS element (Fig. 1c) and the changed expression of OsDREB2A in ONAC066-OE and ONAC066-RNAi plants during drought stress response led us to examine whether ONAC066 regulated directly the expression of OsDREB2A through its binding to NACARS and/or JBS elements in OsDREB2A promoter. Bioinformatics analysis indicated that the 


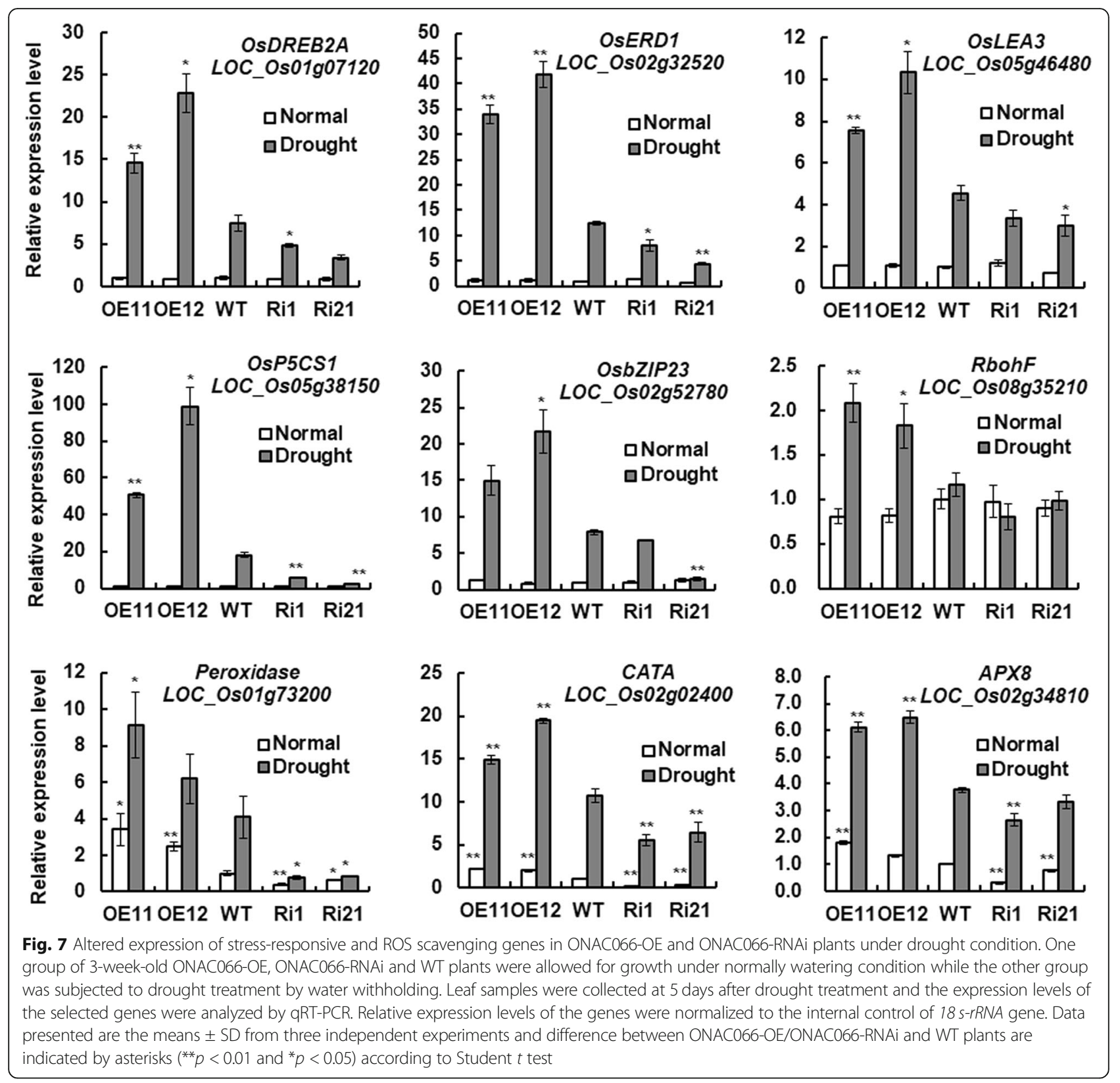

OsDREB2A promoter harbors seven typical NACRS sites and one JBSL site in $1.5 \mathrm{kB}$ region upstream the ATG (Fig. 8a). The DNA binding activity of ONAC066 to the OsDREB2A promoter and JBSL element-containing sequence in the OsDREB2A promoter was examined using $\mathrm{Y} 1 \mathrm{H}$ assays (Fig. 8b). Yeast cells co-transformed with pGADT7-Rec2-ONAC066 and pHis2-pOsDREB2A or pHis2-3 $\times$ JBSL grew normally while yeast cells cotransformed with pGADT7-Rec2 empty vector and pHis2- $p$ OsDREB2A or pHis2-3 $\times$ JBSL failed to grow on $\mathrm{SD} /-$ Trp-Leu-His medium with $50 \mathrm{mM}$ or $100 \mathrm{mM} 3-$ AT (Fig. 8c). We further performed chromatin immunoprecipitation (ChIP) assays to determine the direct interaction between ONAC066 and the promoter of OsDREB2A in planta. ChIP-PCR results showed that a clear band was amplified toward the $\mathrm{P} 2$ probe region but no band was detected toward the P1 and P3 probe regions (Fig. 8d). These results indicate that ONAC066 bound to the P2 probe region, which contained two NACRS and one JBSL site (Fig. 8a), but not bound to P1 probe, which did not harbor NACRS site (Fig. 8a), and P3 probe, which harbored two NACRS sites (Fig. 8a), in OsDREB2A promoter. To confirm whether ONAC066 can bind to the JBSL cis-element in OsDREB2A promoter, recombinant GST-fused ONAC066 protein was purified and examined the binding activity using 


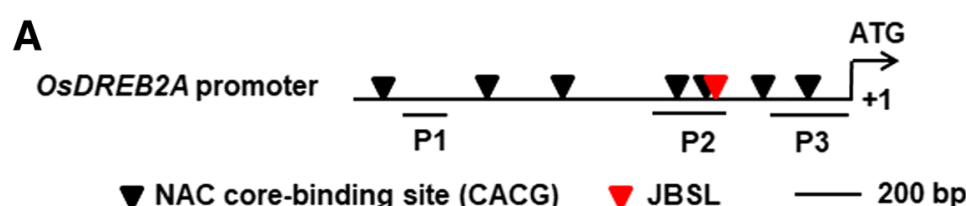

B

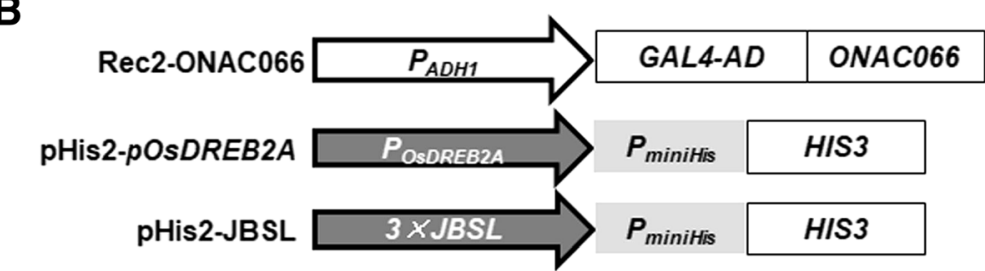

C
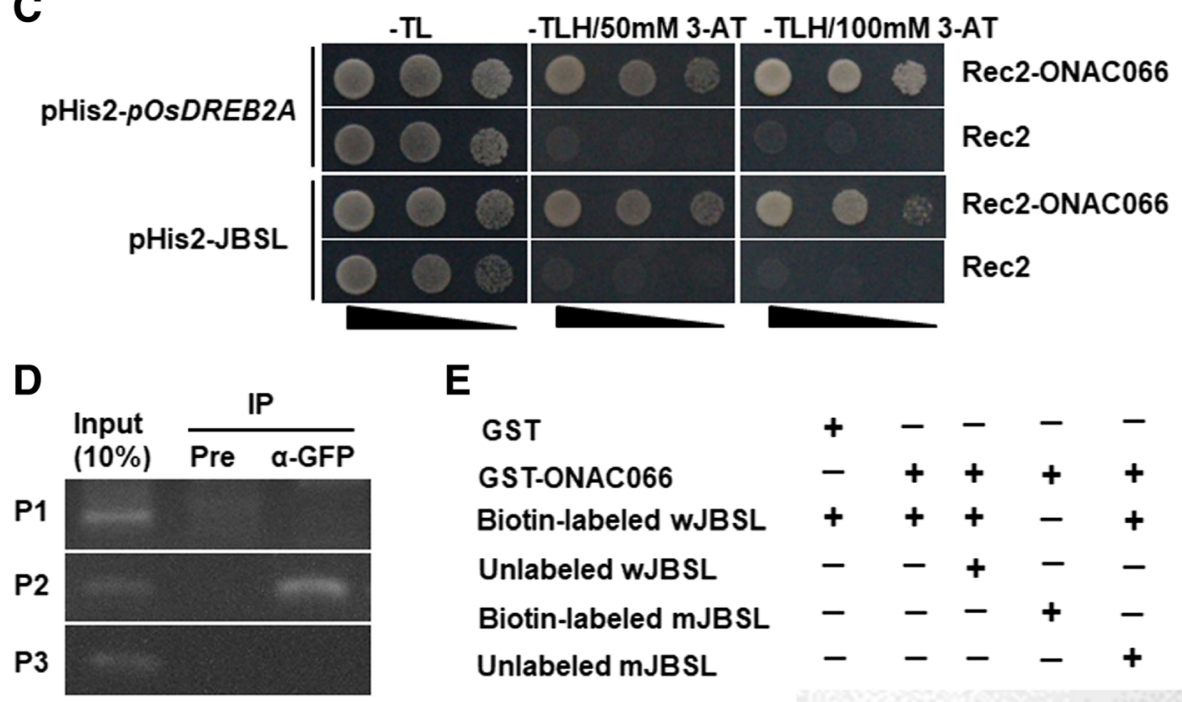

E

$\begin{array}{llllll}\text { GST } & + & - & - & - & - \\ \text { GST-ONAC066 } & - & + & + & + & + \\ \text { Biotin-labeled WJBSL } & + & + & + & - & + \\ \text { Unlabeled wJBSL } & - & - & + & - & - \\ \text { Biotin-labeled mJBSL } & - & - & - & + & - \\ \text { Unlabeled mJBSL } & - & - & - & - & +\end{array}$

Protein-DNA complex

Free probe

Fig. 8 ONAC066 bound to the JBSL cis-element in OSDREB2A promoter. a A diagram showing putative NAC core-binding sequences and JBSL element in the promoter of OSDREB2A. P1, P2 and P3 were the probes used in ChIP-PCR assays. $\mathbf{b}$ Schematic diagram of the different constructs used in ONAC066-pOsDREB2A or ONAC066-pOsDREB2A-JBSL binding assays. c ONAC066 bound to OsDREB2A promoter and JBSL cis-element in OsDREB2A promoter in vivo. Rec2-ONAC066 and Rec2 empty vectors were co-transformed with the reporter vectors pHis-pOsDREB2A or pHispOsDREB2A-JBSL into yeast and co-transformed yeasts were dropped by a series of 10-fold dilutions on medium of SD/-Trp-Leu, SD/-Trp-LeuHis/50 mM 3-AT and SD/-Trp-Leu-His/100 mM 3-AT. Binding activity was estimated according to the growth status of the co-transformed yeasts on different medium at 2 days after plating. $\mathbf{d}$ ONAC066 bound to a JBSL-containing sequence in OsDREB2A promoter. ChIP of ONAC066-GFP transgenic line using GFP antibody (a-GFP) or pre-immune (Pre) serum was performed and precipitated DNA fragments were subject to PCR analysis with OsDREB2A promoter primers. 10\% of chromatin amount before IP was used as positive controls (input) and IP sample with preimmune serum was used as a negative control. e Binding activity of ONAC066 to JBSL cis-element. Biotin-labeled wJBSL and mJBSL probes or biotin-labeled wJBSL probe in combination with unlabeled WJBSL or mJBSL probe were incubated with GST-fused ONAC066 protein or a purified GST preparation as a negative control. Specific protein-DNA complexes and free probes are indicated by the arrowheads on left. Experiments in (c-e) were repeated for three times with similar results

electrophoretic mobility shift assay (EMSA). The EMSA results showed that ONAC066 bound to wJBSL fragment but did not bind to the mutated fragment mJBSL (Fig. 8e). Furthermore, the binding of ONAC066 to labeled wJBSL was suppressed by excessive unlabeled wJBSL but not by excessive unlabeled mJBSL (Fig. 8e). Taken together, these results indicate that ONAC066 can bind to the JBSL cis-element in 
OsDREB2A promoter and thus drive the transcription of OsDREB2A.

\section{Discussion}

NAC TFs have been widely implicated in regulating plant stress responses $[8,12,18,19]$. Among the 151 members in rice NAC family [15-17], nine NAC genes, including ONACO02, ONACO03, ONACO09, ONAC022, ONAC045, ONAC048, ONAC058, ONAC095 and ONAC122, have been shown to play roles in abiotic stress tolerance $[22-34,60]$. In the present study, we demonstrated through functional analyses using overexpression and RNAi-mediated suppression transgenic rice lines that ONAC066, a rice homologue of Arabidopsis AtJUB1 [38, 39, 43], positively regulates drought and oxidative stress tolerance in rice, extending the importance of NAC TFs in plant stress response. Furthermore, biochemical studies revealed that ONAC066 regulates transcription of OsDREB2A through direct binding to a JBSL-containing region in its promoter.

Biochemically, most of the previously identified stressrelated NAC TFs were reported to act as transcription activators, although a few of NAC TFs are transcriptional repressors [61, 62]. We found that ONAC066 has transcription activator activity and this activity is dependent on its C-terminal (Fig. 1a). This is consistent with a common knowledge that NAC TFs have a Cterminal transcriptional activation domain $[22,25,31]$. Importantly, ONAC066 can bind to a canonical NAC recognition cis-element fragment NACRS [14, 63] as well as to a newly identified cis-element JBS [39] in yeasts (Fig. 1b and c). ONAC066 is one of the 63 stressrelated $O N A C$ genes with overlapping expression patterns in rice under various abiotic (e.g., drought and salt) stress conditions [20]. In this study, we verified that the expression of ONAC066 was significantly induced by PEG6000, $\mathrm{NaCl}, \mathrm{H}_{2} \mathrm{O}_{2}$ and ABA (Fig. 2). This is partially supported by the presence of several stress-related cis-elements including two ABREs in the promoter region of the ONAC066 gene (Additional file 1: Figure S1C). Taken together, these observations indicate that ONAC066 is a transcription activator that can respond to multiple abiotic stress factors.

Under drought condition, the ONAC066-OE plants exhibited higher survival ratio and better growth performance while the ONAC066-RNAi plants showed opposite phenotypes (Fig. 3a). These observations demonstrate that ONAC066 is a positive regulator of drought stress tolerance in rice. This is in agreement with the functions of Arabidopsis AtJUB1, tomato SlJUB1 and banana MusaNAC042, which are homologues of ONAC066, in drought stress response [38, 39, 43-45]. The mechanism responsible for the ONAC066-regulated drought stress response can be, at least partially, explained by several physiological and biochemical changes observed in ONAC066-OE and ONAC066-RNAi plants under drought condition. First, changes in WLR and RWC in ONAC066-OE and ONAC066-RNAi plants after drought stress treatment (Fig. 3) may be one of the factors that contribute to the ONAC066-regulated drought stress response. Second, accumulation of compatible solutes such as soluble sugars and free proline is a common phenomenon in response to abiotic stress $[64,65]$. We observed that ONAC066-OE plants accumulated higher levels while ONAC066-RNAi plants accumulated lower levels of free proline and soluble sugars than WT plants under drought condition (Fig. 6a and b). Similar features have been observed in transgenic rice overexpressing ONAC009, ONAC058 and ONAC022 $[28,30,33]$. Altered levels of free proline in ONAC066$\mathrm{OE}$ and ONAC066-RNAi plants under drought condition may be due to the changed expression of OsP5CS1 (Fig. 7), whose overexpression in transgenic rice led to stressinduced accumulation of free proline and increased abiotic stress tolerance [58]. Notably, the contents of free proline and soluble sugars showed an increase in ONAC066-RNAi plants after drought stress treatment, as compared to the non-stressed WT plants (Fig. 6). This imply that other mechanism(s) may compensate the function of ONACO66 and regulate these physiological changes in ONAC066-RNAi plants upon drought stress. Third, the facts of ABA-induced ONAC066 expression (Fig. 2d), changed expression of ABA signaling responsive stressrelated genes (e.g. OsDREB2A [55], OsbZIP23 [59] and OsLEA3 [57]), and affected ABA sensitivity in ONAC066OE and ONAC066-RNAi plants under drought condition (Figs. 5 and 7) demonstrate the involvement of ABA, as a stress hormone, in ONAC066-regulated drought tolerance. This is consistent with previous observations that overexpression of ONAC058 [30], ONACO02 [22], ONACO48 [23] and ONAC022 [33] in transgenic rice led to improved drought tolerance and hypersensitivity to exogenous ABA. However, the involvement of ABAindependent pathway in ONAC066-regulated drought tolerance cannot be ruled out as expression of OsERD1, which is known to be involved in ABA-independent pathway [3], was significantly affected in ONAC066-OE and ONAC066-RNAi plants (Fig. 7).

It has been shown that overexpression of Arabidopsis AtJUB1 confers $\mathrm{H}_{2} \mathrm{O}_{2}$ tolerance [39]. In this study, we also observed that ONAC066-OE plants improved, while ONAC066-RNAi plants attenuated oxidative stress tolerance, as revealed by the leaf damage in $\mathrm{H}_{2} \mathrm{O}_{2}$ and growth performance on MV-containing medium (Fig. 4c and d). Expression ROS generating gene $R b o h F$ was not changed in ONAC066-OE and ONAC066-RNAi plants under normal growth condition; however, expression of ROS scavenging genes $P O D, C A T A$ and APX8 was markedly affected (Fig. 7). These observations indicate that 
modification of ONAC066 expression may affect the ROS scavenging capacity, which in turn account for the changed sensitivity and tolerance to exogenous oxidative stress in ONAC066-OE and ONAC066-RNAi plants. On the other hand, ROS at physiological level is believed to act as signaling molecules that regulate plant adaptation to various stresses while ROS at excessive levels often causes significant damage to plants cells, leading to deleterious effect on stress tolerance [66, 67]. Under water withholding drought condition, relative low level of ROS was detected in ONAC066-OE plants, although expression of ROS generating gene RbohF and ROS scavenging genes $P O D, C A T A$ and $A P X 8$ was simultaneously upregulated (Fig. 7). By contrast, despite the unchanged expression of ROS generating gene RbohF, the expression of ROS scavenging genes POD, CATA and APX8 was significantly downregulated (Fig. 7), leading to excessive accumulation of ROS, in ONAC066-RNAi plants (Fig. 6d). This is consistent with the observations that heterologous expression of AtJUB1 in tomato lowered $\mathrm{H}_{2} \mathrm{O}_{2}$ level and resulted in enhanced drought tolerance while silencing of SIJUB1 increased the accumulation of $\mathrm{H}_{2} \mathrm{O}_{2}$ and led to decreased drought tolerance [45]. Thus, it is likely that ONAC066 assists in regulating cellular ROS homeostasis and thereby regulates drought and oxidative stress tolerance.

It was found that AtJUB1 and SIJUB1 can directly activate transcription of AtDREB2A in Arabidopsis and of SIDREB1 and SIDREB2 in tomato, respectively [39, 45]. The promoter of OSDREB2A, a drought-responsive gene whose overexpression resulted in significant increase of drought stress tolerance in transgenic rice [55], harbors a JBSL cis-element (Fig. 8a). We further verified through $\mathrm{Y} 1 \mathrm{H}, \mathrm{ChIP-PCR}$ and EMSA analyses that ONAC066 binds to the JBSL cis-element in OsDREB2A promoter (Fig. 8b-e). These data, together with the expression patterns of OsDREB2A in ONAC066-OE and ONAC066RNAi plants under drought stress condition (Fig. 7), imply that ONAC066 activates the expression of OsDREB2A through direct binding to the JBSL cis-element in OsDREB2A promoter, further demonstrating the important roles for ONAC066 in drought stress tolerance.

Modification of ONAC066 affected the expression of many stress-responsive and ROS scavenging genes, including OsDREB2A, OsERD1, OsbZIP23, OsP5CS1 and OsAPX8, in ONAC066-OE and ONAC066-RNAi plants during drought stress response (Fig. 7). Except that OsDREB2A promoter contains a JBSL element, OsERD1, OsP5CS1, OsAPX8 and OsbZIP23 also contain sequences similar to JBS/JBSL elements in their promoter regions (2000 bp from start codon) but the similarity is lower than $75 \%$ as compared with the characterized Arabidopsis AtDREB2A JBS element $[39,45]$ and rice OsDREB2A JBSL element (Fig. 8). The remaining 4 genes, including
OsLEA3, OsRbohF, POD and CATA, do not contain JBS/ JBSL elements in their promoters. Therefore, it is most likely that ONAC066 indirectly but not directly regulates the expression of these stress-responsive and ROS scavenging genes during drought stress response. This is partially supported by the observation that the expression levels of most of these stress-responsive and ROS scavenging genes were not affected in ONAC066-OE and ONAC066-RNAi plants under normal unstressed condition (Fig. 7). OsDREB2A plays critical roles in rice drought stress tolerance by acting as an upstream regulator of multiple stress response cascades [55]. The fact that ONAC066 can modulate the OsDREB2A expression by direct binding to the JBSL cis-element in its promoter may imply that OsDREB2A, acting as a master switch, links the function of ONAC066 to the expression of stress-responsive genes upon drought stress. On the other hand, the function of OsDREB2A may be regulated by numerous factors at multiple levels including ONAC066-mediated transcriptional regulations. This may partially explain the phenomena that the expression levels of these stress-responsive and ROS scavenging genes in ONAC066-RNAi plants increased after drought stress treatment, as compared with the WT levels in normal unstressed plants (Fig. 7).

\section{Conclusions}

ONAC066 is a transcription activator that can respond to multiple abiotic stress factors. Functional analyses using overexpression and RNAi-mediated suppression transgenic rice lines demonstrate that ONAC066 acts as a positive regulator of drought and oxidative stress tolerance in rice. Furthermore, ONAC066 activates transcription of OsDREB2A through direct binding to the JBSL cis-element in OSDREB2A promoter. Further characterization of the ONAC066-regulated target genes at genome-wide level will provide new insights into how ONAC066 regulates the drought and oxidative stress tolerance in rice.

\section{Methods \\ Plant materials and stress treatments}

Rice cv. ZH11 (Oryza sativa L. subsp. japonica) was used in all experiments. Rice seeds (Wuhan Biorun Biotechnology Co., Wuhan, China) were sterilized in $0.3 \%$ $\mathrm{NaClO}$ for $15 \mathrm{~min}$, germinated, and cultivated in soil mix or Yoshida's nutrition solution in a growth room with a cycle of $16 \mathrm{~h}$ light at $28^{\circ} \mathrm{C}$ and $8 \mathrm{~h}$ dark at $22^{\circ} \mathrm{C}$. For stress and ABA treatments, three-week-old seedlings were transferred to nutrients solutions containing $20 \%$ (w/v) PEG6000, $100 \mathrm{mM} \mathrm{NaCl}, 100 \mathrm{mM} \mathrm{H}_{2} \mathrm{O}_{2}$ or $50 \mu \mathrm{M}$ $\mathrm{ABA}$, respectively. Leaf samples were collected at indicated time points after treatment, frozen in liquid nitrogen, and stored at $-80^{\circ} \mathrm{C}$ until further analysis. 
Binary vector construction, rice transformation and characterization of ONAC066 transgenic lines The coding sequence of ONACO66 was cloned into pCAMBIA1300s vector to fuse with GFP at C-terminal under the control of Cauliflower mosaic virus (CaMV) 35S promoter, yielding pCAMBIA1300s-ONAC066-GFP. A fragment of $239 \mathrm{bp}$ in 3' divergent region of ONAC066 was amplified and cloned into RNAi vector pTCK303, yielding pTCK303ONAC066. The constructed pCAMBIA1300s-ONAC066GFP and pTCK303-ONAC066 were transformed into ZH11 using Agrobacterium-mediated method [68]. T2 generation of the transgenic lines was assessed for segregation of hygeomycin (Hgr) resistant phenotype on 1/2 MS medium containing $50 \mu \mathrm{g} / \mathrm{L}$ Hgr and lines with 3:1 segregation for Hgrresistant phenotype were chosen as single-copy transgenic lines. Homozygous transgenic lines were screened, according to $100 \%$ Hgr-resistant phenotype, by sowing seeds of T3 generation of the single-copy lines on selective medium. Homozygous single-copy transgenic lines were used for all experiments. Leaf samples were collected from 3-week-old ONAC066-OE and ONAC066-RNAi plants for analyses of the expression level of ONAC066 and the accumulation of ONAC066-GFP fusion protein. Primers used for vector construction are listed in Additional file 4: Table S1.

\section{Subcellular localization assays}

Agrobacteria harboring pCAMBIA1300s-ONAC066GFP and pCAMBIA1300s-GFP empty vector were separately infiltrated into leaves of 3-week-old Nicotiana benthamiana plants expressing a red nuclear marker $\mathrm{RFP}-\mathrm{H} 2 \mathrm{~B}$ protein [54] using $1-\mathrm{mL}$ needless syringes. GFP fluorescence was examined at $48 \mathrm{~h}$ after agroinfiltration and signal was excited at $488 \mathrm{~nm}$ and detected using a $500-530 \mathrm{~nm}$ emission filter under a Zeiss LSM 510 Meta confocal laser scanning microscope (Zeiss, Oberkochen, Germany).

\section{$\mathrm{Y} 1 \mathrm{H}$ assays}

For transcriptional activation assays, the coding sequence and the truncated sequences of ONAC066 were obtained by PCR with different pairs of gene-specific primers (Additional file 4: Table S1) and cloned into pGBKT7 vector to fuse with GAL4 DNA binding domain. The recombinant pGBKT7 vectors, pGBKT7 empty vector (as a negative control) and pGBKT7ONAC022 (as a positive control) [33] were transferred into yeast strain AH109, respectively. The transformed yeast cells were grown on $\mathrm{SD} /-\mathrm{Trp}$ or $\mathrm{SD} /-\mathrm{Trp}$-His medium and incubated for 3 days at $30{ }^{\circ} \mathrm{C}$, followed by addition of 5-bromo-4-chloro-3-indolyl- $\alpha$-D-galactopyranoside $(x-\alpha-g a l)$. Transactivation activity of the fused ONAC066 and its truncated mutants was evaluated according to the growth and production of blue pigments after addition of $\mathrm{x}-\alpha$-gal on $\mathrm{SD} /-\mathrm{Trp}-\mathrm{His} / 5 \mathrm{mM} 3$ AT medium.

For DNA binding assays, the coding sequence of ONAC066 was PCR amplified with a pair of genespecific primers (Additional file 4: Table S1) and fused to the GAL4 activation domain in pGADT7-Rec2, yielding pGADT7-Rec2-ONAC066. Three copies of NACRS, JBS and JBSL sequences in tandems were synthesized and cloned into reporter vector pHis2, yielding pHis2$3 \times$ NACRS, pHis $2-3 \times$ JBS and pHis $2-3 \times$ JBSL. The promoter sequence $(1.0 \mathrm{~Kb}$ upstream from ATG) of OSDREB2A gene was amplified with a pair of specific primers (Additional file 4: Table S1), confirmed by sequencing and cloned into reporter vector $\mathrm{pHis} 2$, yielding pHis2-pOsDREB2A. The pGADT7-Rec2-ONAC066 and pGADT7-Rec2 empty vector were co-transformed along with one of the recombinant pHis2 constructs into yeast strain Y187. The DNA binding activity of ONAC066 was evaluated according to the growth status on SD/-TrpLeu-His/50 mM or $100 \mathrm{mM}$ 3-AT medium.

\section{DNA binding assays}

Coding sequence of ONAC066 was amplified using genespecific primers (Additional file 4: Table S1), inserted into pGEX-6p-1 at EcoRI/SalI sites, and the GST-fused ONAC066 protein was purified using BugBuster GST-Bind Purification Kit (Novagen, Darmstadt, Germany) according to the manufacturer's protocol. A $27 \mathrm{bp}$ JSBL fragment in OsDREB2A promoter wJBSL (5'-GGGAGATGCCGTGACAGGACGCGGTTG-3', the core sequence underlined) and a mutated version mJBSL (5' -GGGAGAAAAAAAGACAGGAAAAAGTTG-3', the mutated nucleotides underlined) were synthesized and labeled with biotin. EMSA was performed using LightShift Chemiluminescent EMSA Kit (Thermo Fisher Scientific, Waltham, MA, USA). Binding reactions were conducted in a total of $20 \mu \mathrm{L}$ volume containing $5 \times$ EMSA buffer, $2 \mu \mathrm{g}$ recombinant ONAC066 protein or GST protein (as a negative control) and $1 \mu \mathrm{L}$ biotinlabeled wJBSL or mJBSL probes. In the competitive reactions, excess unlabeled wJBSLor mJBSL probes was added in excess of 200 times and incubated for $20 \mathrm{~min}$ before addition of the labeled wJBSL probe. The reaction mixtures were separated on 6\% PAGE, transferred onto nitrocellulose membranes and signals from the probes were detected according to the manufacturer's protocol.

\section{Stress tolerance and $A B A$ sensitivity assays}

For drought tolerance assays, 4-week-old ONAC066-OE and WT or ONAC066-RNAi and WT seedlings $(n=30)$ were grown in the same pots with three replicates and were subject to drought stress treatment by water withholding for 7 days, followed by recovery with normally watering for another 2 days. Plants that were green and healthy young leaves after re-watering were regarded as 
survivals and survival ratio was calculated at 2 days after re-watering. For $\mathrm{H}_{2} \mathrm{O}_{2}$ tolerance assays, leaf discs from 3-week-old ONAC066-OE, ONAC066-RNAi and WT plants were incubated in $1 / 2 \mathrm{MS}$ buffer supplemented with $0.5,1$, and $1.5 \% \mathrm{H}_{2} \mathrm{O}_{2}$ or without $\mathrm{H}_{2} \mathrm{O}_{2}$ (as a control) under illumination condition at moderate light intensity $\left(200 \mathrm{mmol} / \mathrm{m}^{2} \mathrm{~s}\right)$. Photographs were taken and samples were collected for analysis of chlorophyll contents at 2 days after treatment. For MV tolerance assays, germinated seeds of ONAC066-OE, ONAC066-RNAi and WT lines were planted on 1/2 MS medium supplemented with $3 \mu \mathrm{M}$ MV or without MV (as a control) and grown for 10 days. Photographs were taken and samples were collected for analysis of chlorophyll contents at 10 days after seed sowing. For ABA sensitivity assays, 60 seeds for each of ONAC066-OE, ONAC066RNAi and WT lines were germinated on 1/2 MS medium supplemented with $2 \mu \mathrm{M}$ or $5 \mu \mathrm{M}$ ABA or without ABA (as a control) in growth room with a cycle of $16 \mathrm{~h}$ light at $28^{\circ} \mathrm{C}$ and $8 \mathrm{~h}$ dark at $22^{\circ} \mathrm{C}$. Photographs were taken and shoot and root length of the seedlings was recorded at 10 days after germination.

\section{Physiological measurements}

WLR and RWC in drought stress tolerance assays were determined according to a previously reported method [69]. Briefly, fully expanded leaves of 3-week-old ONAC066-OE, ONAC066-RNAi and WT plants were detached to record the leaf fresh weight (Wf), turgid leaf weight (Wt) and dry weights (Wd), and WLR and RWC were calculated following the equations: WLR $(\%)=(\mathrm{Wf}-\mathrm{Wt}) /(\mathrm{Wf}-\mathrm{Wd}) \times 100 \%$ and $\mathrm{RWC}(\%)=(\mathrm{Wf}-\mathrm{Wd}) /(\mathrm{Wt}-\mathrm{Wd}) \times 100 \%$. Measurement of chlorophyll content in oxidative stress tolerance assays was performed as described previously [70] using $0.5 \mathrm{~g}$ of leaf samples and the chlorophyll content was calculated according to the formula $\mathrm{Chl}(\mathrm{A}+\mathrm{B})=5.24 \times \mathrm{OD}_{664}+$ $22.24 \times \mathrm{OD}_{648}$.

For analyses of physiological changes and gene expression under drought stress condition, one group of 3week-old ONAC066-OE, ONAC066-RNAi and WT plants were subject to drought treatment by water withholding while the other group of seedlings were grown under normally watered condition. Leaf samples were collected at 5 days after drought treatment and used for further analyses. Contents of free proline was measured by sulphosalicylic acid method [71]. Leaf tissues $(0.2 \mathrm{~g})$ were extracted in $5 \mathrm{ml} \mathrm{3 \%}$ sulfosalicylic acid, and boiled in water for $10 \mathrm{~min}$. After cooling, $2 \mathrm{ml}$ supernatant was incubated with $2 \mathrm{ml}$ glacial acetic acid and $3 \mathrm{ml}$ ninhydrin at $100{ }^{\circ} \mathrm{C}$ for $60 \mathrm{~min}$ and the reaction was terminated on ice bath. After adding $5 \mathrm{ml}$ toluene and incubation at $23^{\circ} \mathrm{C}$ for $24 \mathrm{~h}$, the absorbance was measured at $520 \mathrm{~nm}$ [71]. Content of soluble sugars was determined by the anthrone method [72]. Leaf tissues $(0.5 \mathrm{~g})$ were extracted in $15 \mathrm{ml}$ distilled water by boiling for 20 min with occasional agitation. The supernatant was filtered and brought to a final volume of $100 \mathrm{ml}$ with distilled water. One milliliter of the extract was incubated with $5 \mathrm{~mL}$ anthrone reagent at $95^{\circ} \mathrm{C}$ for $15 \mathrm{~min}$, and then the reaction was terminated on ice, followed by measurement of the absorbance at $620 \mathrm{~nm}$ [72]. In situ detection of $\mathrm{H}_{2} \mathrm{O}_{2}$ and superoxide anion in leaf tissues was performed by DAB staining [73] and NBT staining [74], respectively. Quantitative measurement of $\mathrm{H}_{2} \mathrm{O}_{2}$ in leaf tissues was carried our using $\mathrm{H}_{2} \mathrm{O}_{2}$ detection kit (Nanjing Jiancheng Bioengineering Institute, Nanjing, China) following the manufacturer's instruction.

\section{ChIP and PCR analysis}

ChIP-PCR assays were performed as previously described [75]. Briefly, 3-4 g of 2-week-old ONAC066-OE seedlings was immediately immersed in $60 \mathrm{ml}$ crosslinking buffer $(0.4 \mathrm{M}$ sucrose, $10 \mathrm{mM}$ Tris- $\mathrm{HCl}, \mathrm{pH} 8.0$, $5 \mathrm{mM} \beta$-mercaptoethanol and $1 \%$ formaldehyde) under vacuum for $10 \mathrm{~min}$ and chromatin DNA was extracted. The chromatin DNA was sheared by sonicating into 200-500 bp fragments and pre-cleaned with salmon sperm DNA/protein A-agarose (Millipore, USA) to remove non-specific binding. The sheared DNA was incubated with GFP antibody-conjugated agarose beads (Roche, Switzerland). Agarose beads were washed and the immunoprecipitated DNA was eluted by ChIP elution buffer (1\% SDS, $0.1 \mathrm{M} \mathrm{NaHCO}_{3}$ ). The DNA was dissociated from immunoprecipitation complex and recovered by phenol/chloroform extraction. The chromatins incubated with pre-immune (Pre) serum (GenScript, Nanjing, China) and before immunoprecipitation were used as negative controls and input controls, respectively. PCR was performed using specific primers (Additional file 4: Table S1) and the products were separated on $1.5 \%$ agarose gels and visualized by Goldview staining.

\section{qRT-PCR analyses of gene expression}

Total RNA was extracted using RNA Isolater reagent (Vazyme Biotech, Nanjing, China) according to the manufacturer's protocol. The remaining genomic DNA in RNA samples was removed by treating with genome DNA wiper mix (Vazyme Biotech, Nanjing, China) at $42^{\circ} \mathrm{C}$ for $2 \mathrm{~min}$. First-stranded cDNA was synthesized with $2 \mu \mathrm{g}$ of purified total RNA using reverse transcription system (Vazyme Biotech, Nanjing, China). qRT-PCR was performed on a CFX96 real-time PCR system (BioRad, Hercules, CA, USA) with AceQ qPCR SYBR Green Master Mix (Vazyme Biotech, Nanjing, China). Reactions were run under following conditions: $95^{\circ} \mathrm{C}$ for 5 min, 40 cycles of $95^{\circ} \mathrm{C}$ for $10 \mathrm{~s}, 60^{\circ} \mathrm{C}$ for $15 \mathrm{~s}$ and $72^{\circ} \mathrm{C}$ for $30 \mathrm{~s}$. Rice $18 \mathrm{~s}$-rRNA gene [76] was used as an internal control to normalize data and relative expression 
levels of genes of interest were calculated using the $2^{-\triangle \Delta C T}$ method. Gene-specific primers used in qRT-PCR are listed in Additional file 4: Table S1.

\section{Statistical analysis}

All experiments described were performed in triplicate. Data obtained were statistically analyzed according to the Student's $t$ test and the probability value of $" p<0.05$ or $* p<0.01$ was considered as significant difference.

\section{Additional files}

Additional file 1: Figure S1. Structural and phylogenetic features of ONAC066 and cis-elements in ONAC066 promoter. (A) Alignment of ONAC066 with rice ONAC096, Arabidopsis AtJUB1 and tomato SIJUB1. The conserved NAC domain is underlined with red line and the five highly conserved subdomains $A$ to $E$ are indicated by red boxes. (B) Phylogenetic tree analysis of ONAC066 with other known stressresponsive rice NAC proteins in Phylogeny Group IV. Sequence alignment was performed using Clustal X1.81 program and phylogenic tree was created and visualized using MEGA 5.05. Protein sequences used for alignment are as follow: ANAC042/AtJUB1 (At2g43000), rice ONAC066 (Os03g56580), ONAC096 (Os07g04560), ONAC140 (Os12g43530), ONAC063 (Os08g33910), ONAC049 (Os08g02160), ONAC075 (Os01g66490), ONAC087 (Os05g34600), ONAC022 (Os03g04070), ONAC095 (Os06g51070), ONAC012 (Os05g37080), ONAC059/ENAC1 (Os01g64310), ONAC017/OsNAC111 (Os11g05614), ONAC134 (Os12g05990). (C) Distribution of major stress-related cis-elements in the promoter (1.5 Kb upstream of ATG) of the ONAC066 gene. (JPG $887 \mathrm{~kb}$ )

Additional file 2: Figure S2. Tissue-specific expression of ONAC066. Digital expression of ONAC066 was extracted from public microarray data at NCBI (http://www.ncbi.nlm.nih.gov/geo) under accession number GSE6893). Root, $7 \mathrm{~d}$ seedlings; SAM, up to $0.5 \mathrm{~mm}$, shoot apical meristem and rachis meristem; young inflorescence $\mathrm{P} 1$, up to $3 \mathrm{~cm} ; \mathrm{P} 2,3-5 \mathrm{~cm}$; $\mathrm{P}$, 5-10 cm; P4, 10-15 cm, P5: 15-22 cm, P6: 22-30 cm; seeds S1, 0-2 dap; S2, 3-4 dap; S3, 5-10 dap; S4, 11-20 dap; S5, 21-29 dap. These stage specifications are estimated based on information from previous research [77]. (JPG $351 \mathrm{~kb})$

Additional file 3: Figure S3. Generation and characterization of ONAC066-OE and ONAC066-RNAi transgenic rice lines. (A) Diagrams for overexpression and RNAi constructs. (B) Relative expression of ONAC066 in ONAC066-OE and ONAC066-RNAi transgenic lines. (C) Western blotting detection of ONAC066-GFP fusion protein in ONAC066-OE transgenic lines. (JPG 296 kb)

Additional file 4: Table S1. Primers used in this study. (DOCX 16 kb)

\section{Abbreviations \\ ABA: abscisic acid; ChIP: chromatin immunoprecipitation; DAB: 3, 3'- diaminobenzidine; EMSA: electrophoretic mobility shift assay; JBS: AtJUB1 binding site; JBSL: AtJUB1 JBS-like; MS: Murashige and Skoog medium; MV: methyl viologen; NAC: NAM, ATAF and CUC; NACRS: NAC recognition sequence; NBT: nitroblue tetrazolium; OE: overexpression; qRT- PCR: quantitative reverse transcription-PCR; RNAi: RNA interference; ROS: reactive oxygen species; TF: transcription factor; WT: wild type; $\mathrm{Y} 1 \mathrm{H}$ : yeast one hybrid; $\mathrm{ZH} 11$ : Zhonghua11}

\section{Acknowledgements}

We are grateful to Dr. Michael Goodin (Department of Plant Pathology, University of Kentucky, USA) for providing the H2B-RFP N. benthamiana line.

\section{Authors' contributions}

$X Y, D L$ and $F S$ designed the study; $X Y, H W, J C$ and $Y B$ performed the experiments; $X Y$ and FS wrote the manuscript. All authors read and approved the final manuscript.

\section{Funding}

This study was supported by the National Key Research and Development Program of China (No. 2016YFD0100600) and the National Natural Science Foundation of China (No. 31272028 and No. 31871945). Each of the funding organizations granted the funds based on research proposals and had no influence on the experimental design, data analysis or interpretation, and manuscript writing.

\section{Availability of data and materials}

The datasets supporting the results of this publication are included within the article and its Additional files.

Ethics approval and consent to participate Not applicable.

\section{Consent for publication}

Not applicable.

\section{Competing interests}

The authors declare that they have no competing interests.

Received: 19 November 2018 Accepted: 12 June 2019 Published online: 25 June 2019

\section{References}

1. Zhu JK. Salt and drought stress signal transduction in plants. Annu Rev Plant Biol. 2002;53:247-73.

2. Yamaguchi-Shinozaki K, Shinozaki K. Transcriptional regulatory networks in cellular responses and tolerance to dehydration and cold stresses. Annu Rev Plant Biol. 2006;57:781-803.

3. Shinozaki K, Yamaguchi-Shinozaki K. Gene networks involved in drought stress response and tolerance. J Exp Bot. 2007:58:221-7.

4. Nakashima K, Yamaguchi-Shinozaki K, Shinozaki K. The transcriptional regulatory network in the drought response and its crosstalk in abiotic stress responses including drought, cold, and heat. Front Plant Sci. 2014:5:170

5. Golldack D, Li C, Mohan H, Probst N. Tolerance to drought and salt stress in plants: unraveling the signaling networks. Front Plant Sci. 2014;5:151.

6. Chen L, Song Y, Li S, Zhang L, Zou C, Yu D. The role of WRKY transcription factors in plant abiotic stresses. Biochim Biophys Acta. 2012:1819:120-8.

7. Mizoi J, Shinozaki K, Yamaguchi-Shinozaki K. AP2/ERF family transcription factors in plant abiotic stress responses. Biochim Biophys Acta. 2012;1819: 86-96.

8. Puranik S, Sahu PP, Srivastava PS, Prasad M. NAC proteins: regulation and role in stress tolerance. Trends Plant Sci. 2012:17:369-81.

9. Nuruzzaman M, Sharoni AM, Kikuchi S. Roles of NAC transcription factors in the regulation of biotic and abiotic stress responses in plants. Front Microbiol. 2013;4:248.

10. Lindemose S, O'Shea C, Jensen MK, Skriver K. Structure, function and networks of transcription factors involved in abiotic stress responses. Int J Mol Sci. 2013;14:5842-78.

11. Castilhos G, Lazzarotto F, Spagnolo-Fonini L, Bodanese-Zanettini MH, Margis-Pinheiro M. Possible roles of basic helix-loop-helix transcription factors in adaptation to drought. Plant Sci. 2014;223:1-7.

12. Shao HB, Wang HY, Tang XL. NAC transcription factors in plant multiple abiotic stress responses: progress and prospects. Front Plant Sci. 2015;6:902.

13. Olsen AN, Ernst HA, Leggio LL, Skriver K. NAC transcription factors: structurally distinct, functionally diverse. Trends Plant Sci. 2005:10:79-87.

14. Tran LS, Nakashima K, Sakuma Y, Simpson SD, Fujita Y, Maruyama K, Fujita M, Seki M, Shinozaki K, Yamaguchi-Shinozaki K. Isolation and functional analysis of Arabidopsis stress-inducible NAC transcription factors that bind to a drought-responsive cis-element in the early responsive to dehydration stress 1 promoter. Plant Cell. 2004;16:2481-98.

15. Ooka H, Satoh K, Doi K, Nagata T, Otomo Y, Murakami K, Matsubara K, Osato N, Kawai J, Carninci P, Hayashizaki Y, Suzuki K, Kojima K, Takahara Y, Yamamoto K, Kikuchi S. Comprehensive analysis of NAC family genes in Oryza sativa and Arabidopsis thaliana. DNA Res. 2003;10:239-47.

16. Fang Y, You J, Xie K, Xie W, Xiong L. Systematic sequence analysis and identification of tissue-specific or stress-responsive genes of NAC transcription factor family in rice. Mol Gen Genomics. 2008;280:547-63. 
17. Nuruzzaman M, Manimekalai R, Sharoni AM, Satoh K, Kondoh H, Ooka H, Kikuchi S. Genome-wide analysis of NAC transcription factor family in rice. Gene. 2010;465:30-44.

18. Nuruzzaman M, Sharoni AM, Satoh K, Moumeni A, Venuprasad R, Serraj R, Kumar A, Leung H, Attia K, Kikuchi S. Comprehensive gene expression analysis of the NAC gene family under normal growth conditions, hormone treatment, and drought stress conditions in rice using near-isogenic lines (NILs) generated from crossing Aday selection (drought tolerant) and IR64. Mol Gen Genomics. 2012;287:389-410.

19. Nakashima K, Takasaki H, Mizoi J, Shinozaki K, Yamaguchi-Shinozaki K. NAC transcription factors in plant abiotic stress responses. Biochim Biophys Acta. 2012;1819:97-103.

20. Sun LJ, Huang L, Hong YB, Zhang HJ, Song FM, Li DY. Comprehensive analysis suggests overlapping expression of rice ONAC transcription factors in abiotic and biotic stress responses. Int J Mol Sci. 2015;16:4306-26.

21. Nuruzzaman M, Sharoni AM, Satoh K, Karim MR, Harikrishna JA, Shimizu T, Sasaya T, Omura T, Haque MA, Hasan SM, Ahmad A Kikuchi S. NAC transcription factor family genes are differentially expressed in rice during infections with Rice dwarf virus, Rice blackstreaked dwarf virus, Rice grassy stunt virus, Rice ragged stunt virus, and Rice transitory yellowing virus. Front Plant Sci. 2015;6:676.

22. Hu H, Dai M, Yao J, Xiao B, Li X, Zhang Q, Xiong L. Overexpressing a NAM, ATAF, and CUC (NAC) transcription factor enhances drought resistance and salt tolerance in rice. Proc Natl Acad Sci U S A. 2006:103:12987-92.

23. Hu HH, You J, Fang YJ, Zhu XY, Qi ZY, Xiong LZ. Characterization of transcription factor gene SNAC2 conferring cold and salt tolerance in rice. Plant Mol Biol. 2008;67:169-81.

24. Zheng X, Chen B, Lu G, Han B. Overexpression of a NAC transcription factor enhances rice drought and salt tolerance. Biochem Biophys Res Commun. 2009;379:985-9.

25. Takasaki H, Maruyama K, Kidokoro S, Ito Y, Fujita Y, Shinozaki K, YamaguchiShinozaki K, Nakashima K. The abiotic stress-responsive NAC-type transcription factor OsNAC5 regulates stress-inducible genes and stress tolerance in rice. Mol Gen Genomics. 2010;284:173-83.

26. Jeong JS, Kim YS, Baek KH, Jung H, Ha SH, Do Choi Y, Kim M, Reuzeau C, Kim JK. Root-specific expression of OsNAC10 improves drought tolerance and grain yield in rice under field drought conditions. Plant Physiol. 2010;153:185-97.

27. Jeong JS, Kim YS, Redillas MC, Jang G, Jung H, Bang SW, Choi YD, Ha SH, Reuzeau C, Kim JK. OsNAC5 overexpression enlarges root diameter in rice plants leading to enhanced drought tolerance and increased grain yield in the field. Plant Biotechnol J. 2013;11:101-14.

28. Song SY, Chen Y, Chen J, Dai XY, Zhang WH. Physiological mechanisms underlying OsNAC5-dependent tolerance of rice plants to abiotic stress. Planta. 2011;234:331-45.

29. Redillas MC, Jeong JS, Kim YS, Jung H, Bang SW, Choi YD, Ha SH, Reuzeau C, Kim JK. The overexpression of OsNAC9 alters the root architecture of rice plants enhancing drought resistance and grain yield under field conditions. Plant Biotechnol J. 2012;10:792-805.

30. Chen X, Wang Y, Lv B, Li J, Luo L, Lu S, Zhang X, Ma H, Ming F. The NAC family transcription factor OsNAP confers abiotic stress response through the ABA pathway. Plant Cell Physiol. 2014;55:604-19.

31. Liang C, Wang Y, Zhu Y, Tang J, Hu B, Liu L, Ou S, Wu H, Sun X, Chu J, Chu C. OsNAP connects abscisic acid and leaf senescence by fine-tuning abscisic acid biosynthesis and directly targeting senescence-associated genes in rice. Proc Natl Acad Sci U S A. 2014;111:10013-8.

32. Fang $Y$, Liao $K$, Du H, Xu Y, Song H, Li X, Xiong L. A stress-responsive NAC transcription factor SNAC3 confers heat and drought tolerance through modulation of reactive oxygen species in rice. J Exp Bot. 2015;66:6803-17.

33. Hong $Y$, Zhang $H$, Huang L, Li D, Song F. Overexpression of a stressresponsive NAC transcription factor gene ONAC022 improves drought and salt tolerance in rice. Front Plant Sci. 2016;7:4.

34. Huang $L$, Hong $Y$, Zhang $H$, Li D, Song F. Rice NAC transcription factor ONAC095 plays opposite roles in drought and cold stress tolerance. BMC Plant Biol. 2016;16:203.

35. Lee DK, Chung PJ, Jeong JS, Jang G, Bang SW, Jung H, Kim YS, Ha SH, Choi YD, Kim JK. The rice OsNAC6 transcription factor orchestrates multiple molecular mechanisms involving root structural adaptions and nicotianamine biosynthesis for drought tolerance. Plant Biotechnol J. 2017;15:754-64.

36. Tran LS, Nishiyama R, Yamaguchi-Shinozaki K, Shinozaki K. Potential utilization of NAC transcription factors to enhance abiotic stress tolerance in plants by biotechnological approach. GM Crops. 2010;1:32-9.
37. $\mathrm{Hu} \mathrm{H}$, Xiong L. Genetic engineering and breeding of drought-resistant crops. Annu Revi Plant Biol. 2014:65:715-41.

38. Shahnejat-Bushehri S, Mueller-Roeber B, Balazadeh S. Arabidopsis NAC transcription factor JUNGBRUNNEN1 affects thermomemory-associated genes and enhances heat stress tolerance in primed and unprimed conditions. Plant Signal Behav. 2012;7:1518-21.

39. Wu A, Allu AD, Garapati $P$, Siddiqui H, Dortay H, Zanor MI, Asensi-Fabado MA, Munne-Bosch S, Antonio C, Tohge T, Fernie AR, Kaufmann K, Xue GP, Mueller-Roeber B, Balazadeh S. JUNGBRUNNEN1, a reactive oxygen speciesresponsive NAC transcription factor, regulates longevity in Arabidopsis. Plant Cell. 2012;24:482-506

40. Ebrahimian-Motlagh S, Ribone PA, Thirumalaikumar VP, Allu AD, Chan RL, Mueller-Roeber B, Balazadeh S. JUNGBRUNNEN1 confers drought tolerance downstream of the HD-zip I transcription factor AtHB13. Front Plant Sci. 2017:8:2118.

41. Shahnejat-Bushehri S, Nobmann B, Devi Allu A, Balazadeh S. JUB1 suppresses Pseudomonas syringae-induced defense responses through accumulation of DELLA proteins. Plant Signal Behav. 2016;11:e1181245.

42. Shahnejat-Bushehri S, Tarkowska D, Sakuraba Y, Balazadeh S. Arabidopsis NAC transcription factor JUB1 regulates GA/BR metabolism and signalling. Nat Plants. 2016;2:16013.

43. Ebrahimian-Motlagh S, Ribone PA, Thirumalaikumar VP, Allu AD, Chan RL, Mueller-Roeber B, Balazadeh S. JUNGBRUNNEN1 confers frought tolerance downstream of the HD-zip I transcription factor AtHB13. Front Plant Sci. 2017:8:2118.

44. Tak H, Negi S, Ganapathi TR. Banana NAC transcription factor MusaNAC042 is positively associated with drought and salinity tolerance. Protoplasma. 2017:254:803-16.

45. Thirumalaikumar VP, Devkar V, Mehterov N, Ali S, Ozgur R, Turkan I, MuellerRoeber B, Balazadeh S. NAC transcription factor JUNGBRUNNEN1 enhances drought tolerance in tomato. Plant Biotechnol J. 2018;16:354-66.

46. Lescot M, Dehais $P$, Thijs $G$, Marchal K, Moreau $Y$, Van de Peer $Y$, Rouze P, Rombauts S. PlantCARE, a database of plant cis-acting regulatory elements and a portal to tools for in silico analysis of promoter sequences. Nucleic Acids Res. 2002;30:325-7.

47. Busk PK, Pages M. Regulation of abscisic acid-induced transcription. Plant Mol Biol. 1998;37:425-35.

48. Rouster J, Leah R, Mundy J, Cameron-Mills V. Identification of a methyl jasmonate-responsive region in the promoter of a lipoxygenase 1 gene expressed in barley grain. Plant J. 1997;11:513-23.

49. Ohme-Takagi $M$, Shinshi $H$. Ethylene-inducible DNA binding proteins that interact with an ethylene-responsive element. Plant Cell. 1995;7:173-82.

50. Goldsbrough AP, Albrecht H, Stratford R. Salicylic acid-inducible binding of a tobacco nuclear protein to a $10 \mathrm{bp}$ sequence which is highly conserved amongst stress-inducible genes. Plant J. 1993;3:563-71.

51. Salazar M, Gonzalez E, Casaretto JA, Casacuberta JM, Ruiz-Lara S. The promoter of the TLC1.1 retrotransposon from Solanum chilense is activated by multiple stress-related signaling molecules. Plant Cell Rep. 2007;26:1861-8.

52. Diaz-De-Leon F, Klotz KL, Lagrimini LM. Nucleotide sequence of the tobacco (Nicotiana tabacum) anionic peroxidase gene. Plant Physiol. 1993;101:1117-8.

53. Scharf KD, Rose S, Zott W, Schoffl F, Nover L. Three tomato genes code for heat stress transcription factors with a region of remarkable homology to the DNA-binding domain of the yeast HSF. EMBO J. 1990;9:4495-501.

54. Chakrabarty R, Banerjee R, Chung SM, Farman M, Citovsky V, Hogenhout SA, Tzfira T, Goodin M. PSITE vectors for stable integration or transient expression of autofluorescent protein fusions in plants: probing Nicotiana benthamiana-virus interactions. Mol Plant-Microbe Interact. 2007;20:740-50.

55. Dubouzet JG, Sakuma Y, Ito Y, Kasuga M, Dubouzet EG, Miura S, Seki M, Shinozaki K, Yamaguchi-Shinozaki K. OsDREB genes in rice, Oryza sativa L., encode transcription activators that function in drought-, high-salt- and cold-responsive gene expression. Plant J. 2003;33:751-63.

56. Kiyosue T, Yamaguchi-Shinozaki K, Shinozaki K. Cloning of cDNAs for genes that are early-responsive to dehydration stress (ERDs) in Arabidopsis thaliana L.: identification of three ERDs as HSP cognate genes. Plant Mol Biol. 1994; 25:791-8.

57. Xiao B, Huang $Y$, Tang $N$, Xiong L. Over-expression of a LEA gene in rice improves drought resistance under the field conditions. Theor Appl Genet. 2007;115:35-46.

58. Xiong H, Li J, Liu P, Duan J, Zhao Y, Guo X, Li Y, Zhang H, Ali J, Li Z. Overexpression of OSMYB48-1, a novel MYB-related transcription factor, enhances drought and salinity tolerance in rice. PLoS One. 2014;9:e92913. 
59. Xiang Y, Tang N, Du H, Ye H, Xiong L. Characterization of OsbZIP23 as a key player of the basic leucine zipper transcription factor family for conferring abscisic acid sensitivity and salinity and drought tolerance in rice. Plant Physiol. 2008;148:1938-52.

60. Heller FO, Resch A. Nucleic structure in relation to functional change in the stomata of Vicia faba. Planta. 1967;75:243-52.

61. Kim HS, Park BO, Yoo JH, Jung MS, Lee SM, Han HJ, Kim KE, Kim SH, Lim CO, Yun DJ, et al. Identification of a calmodulin-binding NAC protein as a transcriptional repressor in Arabidopsis. J Biol Chem. 2007;282:36292-302.

62. Lee MH, Jeon HS, Kim HG, Park OK. An Arabidopsis NAC transcription factor NAC4 promotes pathogen- induced cell death under negative regulation by microRNA164. New Phytol. 2017;214:343-60.

63. Xie $\mathrm{Q}$, Frugis $\mathrm{G}$, Colgan $\mathrm{D}$, Chua NH. Arabidopsis NAC1 transduces auxin signal downstream of TIR1 to promote lateral root development. Genes Dev. 2000;14:3024-36.

64. Garg AK, Kim JK, Owens TG, Ranwala AP, Choi YD, Kochian LV, Wu RJ. Trehalose accumulation in rice plants confers high tolerance levels to different abiotic stresses. Proc Natl Acad Sci U S A. 2002;99:15898-903.

65. Liu J, Zhu JK. Proline accumulation and salt-stress-induced gene expression in a salt-hypersensitive mutant of Arabidopsis. Plant Physiol. 1997;114:591-6.

66. You J, Chan ZL. ROS regulation during abiotic stress responses in crop plants. Front Plant Sci. 2015;6:1092.

67. Choudhury S, Panda P, Sahoo L, Panda SK. Reactive oxygen species signaling in plants under abiotic stress. Plant Signal Behav. 2013;8:e23681.

68. Ozawa K. A high-efficiency agrobacterium-mediated transformation system of rice (Oryza sativa $\mathrm{L}$ ). Methods Mol Biol. 2012;847:51-7.

69. Schonfeld MA, Johnson RC, Carver BF, Mornhinweg DW. Water relations in winter wheat as drought resistance indicators. Crop Sci. 1988;28:526-31.

70. Lichtenthaler HK, Buschmann C. Chlorophyll fluorescence spectra of green bean leaves. J Plant Physiol. 1987;129:137-47.

71. Troll W, Lindsley J. A photometric method for the determination of proline. J Biol Chem. 1955;215:655-60.

72. Morris DL. Quantitative determination of carbohydrates with Dreywood's anthrone reagent. Science. 1948;107:254-5.

73. Thordal-Christensen $\mathrm{H}$, Zhang Z, Wei $\mathrm{Y}$, Collinge DB. Subcellular localization of $\mathrm{H}_{2} \mathrm{O}_{2}$ in plants. $\mathrm{H}_{2} \mathrm{O}_{2}$ accumulation in papillae and hypersensitive response during the barley-powdery mildew interaction. Plant J. 2010;11: 1187-94.

74. Doke N. Involvement of superoxide anion generation in the hypersensitive response of potato tuber tissues to infection with an incompatible race of Phytophthora infestans and to the hyphal wall components. Physiol Plant Pathol. 1983;23:345-57.

75. Haring M, Offermann S, Danker T, Horst I, Peterhansel C, Stam M. Chromatin immunoprecipitation: optimization, quantitative analysis and data normalization. Plant Methods. 2007;3:11

76. Jain M, Nijhawan A, Tyagi AK, Khurana JP. Validation of housekeeping genes as internal control for studying gene expression in rice by quantitative realtime PCR. Biochem Biophys Res Commun. 2006:345:646-51.

77. Itoh J, Nonomura K, Ikeda K, Yamaki S, Inukai Y, Yamagishi H, Kitano H, Nagato Y. Rice plant development: from zygote to spikelet. Plant Cell Physiol. 2005;46:23-47.

\section{Publisher's Note}

Springer Nature remains neutral with regard to jurisdictional claims in published maps and institutional affiliations.

Ready to submit your research? Choose BMC and benefit from:

- fast, convenient online submission

- thorough peer review by experienced researchers in your field

- rapid publication on acceptance

- support for research data, including large and complex data types

- gold Open Access which fosters wider collaboration and increased citations

- maximum visibility for your research: over $100 \mathrm{M}$ website views per year

At BMC, research is always in progress.

Learn more biomedcentral.com/submissions 